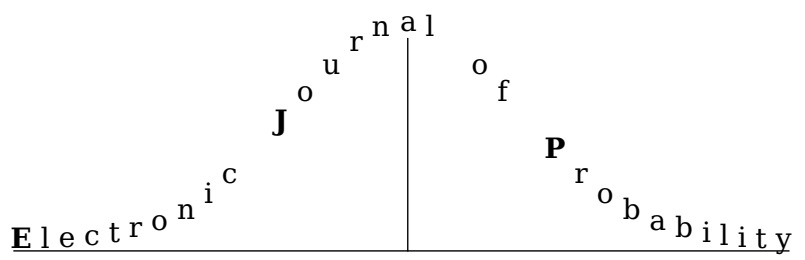

Electron. J. Probab. 26 (2021), article no. 66, 1-36.

ISSN: 1083-6489 https://doi.org/10.1214/21-EJP622

\title{
Erratic behavior for 1-dimensional random walks in a Liouville quasi-periodic environment*
}

\author{
Dmitry Dolgopyat ${ }^{\dagger} \quad$ Bassam Fayad $^{\ddagger} \quad$ Maria Saprykina $^{\S}$
}

\begin{abstract}
We show that one-dimensional random walks in a quasi-periodic environment with Liouville frequency generically have an erratic statistical behavior. In the recurrent case we show that neither quenched nor annealed limit theorems hold and both drift and variance exhibit wild oscillations, being logarithmic at some times and almost linear at other times. In the transient case we show that the annealed Central Limit Theorem fails generically. These results are in stark contrast with the Diophantine case where the Central Limit Theorem with linear drift and variance was established by Sinai.
\end{abstract}

Keywords: random walks in random environment; random walks in random potential; Liouville phenomena; localization.

MSC2020 subject classifications: Primary 60K37; 60F15, Secondary 37C05; 37A45.

Submitted to EJP on May 16, 2020, final version accepted on April 3, 2021.

\section{Introduction}

\subsection{Quasiperiodic random walks}

Let $C^{\infty}(\mathbb{T},(0,1))$ be the set of smooth functions from the standard torus $\mathbb{T}=\mathbb{R} / \mathbb{Z}$ to $(0,1)$ (these can be identified with smooth 1-periodic functions from $\mathbb{R}$ to $(0,1)$ ). Each triple $(\mathfrak{p}, \alpha, x)$, where $\mathfrak{p} \in C^{\infty}(\mathbb{T},(0,1)), \alpha \in(0,1)$ and $x \in \mathbb{T}$ defines a sequence of numbers $\mathrm{p}(j)=\mathfrak{p}(x+j \alpha)$ for all $j \in \mathbb{Z}$. The sequence $(\mathrm{p}(j))_{j \in \mathbb{Z}}$ will be called the quasi-periodic environment defined by $(\mathfrak{p}, \alpha, x)$, or just environment $(\mathfrak{p}, \alpha, x)$.

Consider the nearest neighbor random walk $\left(Z_{t}\right)_{t \in \mathbb{N}}$ on the one dimensional lattice $\mathbb{Z}$, given by $Z_{0}=0$, and for $t \in \mathbb{N}$

$$
\mathbb{P}_{x}\left(Z_{t+1}=k+1 \mid Z_{t}=k\right)=\mathfrak{p}(x+k \alpha), \quad \mathbb{P}_{x}\left(Z_{t+1}=k-1 \mid Z_{t}=k\right)=\mathfrak{q}(x+k \alpha),
$$

${ }^{*}$ Maria Saprykina was supported in part by the Swedish Research Council, VR 2015-04012. Dmitry Dolgopyat was supported in part by the NSF. Bassam Fayad was supported in part by ANR-15-CE40-0001 grant and Knut and Alice Wallenberg foundation, grant KAW 2016.0403.

${ }^{\dagger}$ University of Maryland, USA. E-mail: dolgop@umd .edu

${ }^{\ddagger}$ CNRS UMR7586 - IMJ-PRG Paris, France. E-mail: bassam. fayad@imj - prg. fr

${ }^{\S}$ Institute för Matematik, KTH Stockholm, Sweden. E-mail: masha@kth. se 
Random walks in a Liouville quasi-periodic environment

where $\mathfrak{q}(x)=1-\mathfrak{p}(x)$, and where we stressed the dependence on $x$ with the notation $\mathbb{P}_{x}$.

Following [25], one can also define a related Markov process $\left(X_{t}\right)_{t \in \mathbb{N}}$ on $\mathbb{T}$ :

$$
X_{t}=X_{0}+Z_{t} \alpha \bmod 1, \quad X_{0}=x .
$$

When $\alpha \notin \mathbb{Q}$, we call $\left(Z_{t}\right)_{t \in \mathbb{N}}$ a one-dimensional random walk in quasi-periodic environment, or for short a quasi-periodic walk. The behavior of these walks has many similarities with that of the classical random walks in a random environment, and yet many differences. We will present the statements in different contexts, in particular, quenched and annealed limit laws, as defined below.

Definition 1.1. We use the notation $\mathbb{P}_{x}$ for the distribution of the paths of $\left(Z_{t}\right)_{t \in \mathbb{N}}$ defined in (1.1), when $x \in \mathbb{T}$ is fixed, and $\mathbb{P}_{\text {Leb }}$ for the distribution of the paths of $\left(Z_{t}\right)_{t \in \mathbb{N}}$ when $x$ is uniformly distributed on $\mathbb{T}$ with respect to the Haar measure Leb on $\mathbb{T}$. The notation $\mathbb{E}_{x}$ is reserved for the expectation under the probability $\mathbb{P}_{x}$, while $\mathbb{E}_{\text {Leb }}$ is used for the expectation under $\mathbb{P}_{\text {Leb }}$. Similarly, we use the notations $\operatorname{Var}_{x}$ and $\operatorname{Var}_{\text {Leb }}$ to denote variances for $\mathbb{P}_{x}$ and $\mathbb{P}_{\text {Leb. }}$. The same notations will be used for the distribution of the paths of $\left(X_{t}\right)_{t \in \mathbb{N}}$ defined in (1.2).

Definition 1.2 (Quenched and Annealed limit theorems). Consider a quasi-periodic environment defined by a triple $(\mathfrak{p}, \alpha, x)$ where $\mathfrak{p} \in C^{\infty}(\mathbb{T},(0,1)), \alpha \notin \mathbb{Q}$ and $x \in \mathbb{T}$, and let $\left(Z_{t}\right)_{t \in \mathbb{N}}$ be the random walk defined as in (1.1). When $x \in \mathbb{T}$ is fixed, we say that the walk $\left(Z_{t}\right)_{t \in \mathbb{N}}$ satisfies a quenched limit theorem if there exist sequences $\left(b_{t}(x)\right)_{t \in \mathbb{N}}$ and $\left(\sigma_{t}(x)\right)_{t \in \mathbb{N}}$ and a proper distribution $\mathcal{D}_{x}(\cdot)$ such that for any $z \in \mathbb{R}$

$$
\lim _{t \rightarrow \infty} \mathbb{P}_{x}\left(Z_{t}-b_{t}(x)<\sigma_{t}(x) z\right)=\mathcal{D}_{x}(z) .
$$

We say that the walk satisfies an annealed limit theorem if there exist sequences $\left(b_{t}\right)_{t \in \mathbb{N}}$ and $\left(\sigma_{t}\right)_{t \in \mathbb{N}}$ and a proper distribution $\mathcal{D}(\cdot)$ such that for any $z \in \mathbb{R}$

$$
\lim _{t \rightarrow \infty} \mathbb{P}_{\mathrm{Leb}}\left(Z_{t}-b_{t}<\sigma_{t} z\right)=\mathcal{D}(z) .
$$

In analogy with the drift of simple random walks, we call $b_{t}(x)$ or $b_{t}$ the drift at time $t$.

We will see that the notion of symmetry is very important in distinguishing different types of quasi-periodic walks. Following [25], we adopt the following definition.

Definition 1.3 (Symmetric and asymmetric walks). Given an environment defined by $(\mathfrak{p}, \alpha, x)$, we call $\left(Z_{t}\right)_{t \in \mathbb{N}}$ defined as in (1.1) a symmetric quasi-periodic walk if $\mathfrak{p}$ satisfies

$$
\int_{\mathbb{T}} \ln \mathfrak{p}(x) d x=\int_{\mathbb{T}} \ln \mathfrak{q}(x) d x .
$$

Otherwise we say that the walk is asymmetric. We denote by $\mathcal{P} \subset C^{\infty}(\mathbb{T},(0,1))$ the set of functions satisfying the symmetry condition (1.3), and by $\mathcal{P}^{c}=C^{\infty}(\mathbb{T},(0,1)) \backslash \mathcal{P}$-the set of asymmetric walks.

Note that in general, even if $\mathfrak{p} \in \mathcal{P}$, the sequence $(\mathfrak{p}(x+k \alpha))_{k \in \mathbb{Z}}$ does not exhibit any symmetry. The effect of the symmetry condition above is the result of the averaging effect, which comes from the equidistribution of any orbit of an irrational rotation on the circle. It is a classical fact in dynamical systems that the effectiveness of the equidistribution of the orbits of an irrational rotation of the circle is determined by the arithmetic properties of its angle $\alpha$.

Recall that $\alpha \in \mathbb{R}$ is said to be Diophantine (denoted $\alpha \in \operatorname{DC}(\gamma, \tau)$ ) if there exists $\gamma>0$ and $\tau \geq 0$ such that for any $(p, q) \in \mathbb{Z} \times \mathbb{N}^{*}$

$$
\left|\alpha-\frac{p}{q}\right| \geq \frac{\gamma}{q^{2+\tau}} .
$$


Random walks in a Liouville quasi-periodic environment

An irrational real number that is not Diophantine is called Liouville.

An elementary but noticeable fact of number theory is that Liouville numbers form a dense $G^{\delta}$ set of $\mathbb{R}$, while the Lebesgue measure of this set is zero.

We now introduce a short hand notation that will help simplify the exposition.

Definition 1.4 (Diophantine and Liouville walks). We call $\left(Z_{t}\right)_{t \in \mathbb{N}}$ defined as in (1.1) with $\alpha \in \mathrm{DC}$ a Diophantine walk. If $\alpha \notin \mathbb{Q} \cup \mathrm{DC}$, we call the walk Liouville.

Because the equidistribution of Diophantine rotations is more effective than that of the Liouville ones, the averaging on the medium due to quasi-periodicity is more effective in the case of Diophantine quasi-periodic environment. As a result, they behave similarly to simple random walks, as was established by Sinai in [25]. Namely, they have linear (possibly null) drift and satisfy the Central Limit Theorem. The precise statements for Diophantine walks will be recalled in §2.1. By contrast, nothing was known for Liouville environments beyond the results which hold in any uniquely ergodic environment.

In this paper we study the Liouville case. We show that for any given Liouville $\alpha$ the walk $\left(Z_{t}\right)_{t \in \mathbb{N}}$ defined by (1.1) with a generic $\mathfrak{p} \in C^{\infty}(\mathbb{T},(0,1))$ has a very erratic statistical behavior. By generic we mean of first category for the $C^{\infty}$ topology. Since a generic irrational $\alpha$ is Liouville, our results imply the erratic behavior for one-dimensional random walks in a generic quasi-periodic environment. What we mean by erratic, is that at different times scales the walk $\left(Z_{t}\right)_{t \in \mathbb{N}}$ behaves very differently (think of a walk that drifts almost linearly for a subsequence of times $\left(t_{n}\right)_{n \in \mathbb{N}}$, while for another subsequence $\left(t_{n}^{\prime}\right)_{n \in \mathbb{N}}$ it will be localized logarithmically around the origin).

Our main results can be summarized as follows. For symmetric random walks we show that the following behavior is generic:

- The spread of the walk (as measured, for example, by standard deviation) oscillates wildly. Sometimes the walk is localized at a logarithmic scale while at other times the variance grows faster than $t^{1-\varepsilon}$. At the latter scales the walk bypasses both $-t^{1-\varepsilon}$ and $t^{1-\varepsilon}$ with probability larger than 0.1 .

- The drift of the walk oscillates wildly: sometimes it is larger than $t^{1-\varepsilon}$, sometimes it is smaller than $-t^{1-\varepsilon}$, sometimes it is of order 1 .

- The walk does not satisfy neither an annealed nor a quenched limit theorem: the set of limit distributions includes the normal distribution as well as a distribution with atoms.

We will also show that

- A one-dimensional random walk in a generic asymmetric quasi-periodic environment does not have an annealed limit law.

The precise statements of the results outlined above are contained in §2.2.

Plan of the paper and outline of the proofs. Section 2 contains all the main statements and a review of related resutls from the literature. Quasi-periodic Diophantine environments are discussed in §2.1. \$2.2 contains the precise statements about Liouville walks. It turns out that their behavior is quite different from the Diophantine walks, and is, in fact, quite similar to the walks in a generic deterministic elliptic environment that we define and discuss in \$2.3. The Liouville walks are more erratic than the walks in independent random environments that we briefly review in $\$ 2.5$. Several open questions motivated by the present work are discussed in $\$ 2.4$.

The main technical tool in the study of one dimensional random walks in a fixed environment given by transition probabilities $\mathrm{p}_{j}, j \in \mathbb{Z}$ and $\mathrm{q}_{j}=1-\mathrm{p}_{j}$, is the martingale (3.9). The important quantities that are involved in this martingale, and that determine 
the behavior of the walk, are the sums

$$
\Sigma(n)= \begin{cases}\sum_{j=1}^{n} \ln \mathrm{q}_{j}-\ln \mathrm{p}_{j} & \text { if } n \geq 1, \\ 0, & \text { if } n=0 \\ \sum_{j=n+1}^{0} \ln \mathrm{p}_{j}-\ln \mathrm{q}_{j} & \text { if } n \leq-1 .\end{cases}
$$

The function $n \mapsto \Sigma(n)$ is known as the potential. A direct inspection shows that if $\mathrm{p}_{j}>\mathrm{q}_{j}$ for all $j$ in some interval $I \subset \mathbb{Z}$ then $\Sigma$ is decreasing on $I$, while if $\mathrm{q}_{j}>\mathrm{p}_{j}$ for all $j \in I$ then $\Sigma$ is increasing on $I$. Thus, the guiding intuition is that the walker tends to go downwards on the graph of $\Sigma$ and spends a lot of time near local minima of the potential. The study of the potential plays a crucial role in the study of random walks on $\mathbb{Z}$ starting with the pioneering work of Sinai [24] and it is also central in the present paper.

The reason for the strong difference of behavior between Diophantine and Liouville walks. In the case of quasi-periodic walks defined by some $\mathfrak{p} \in C^{\infty}(\mathbb{T},(0,1))$ as in (1.1), both the transition probabilities $\mathrm{p}_{x, j}=\mathfrak{p}(x+j \alpha)$ and the sums $\Sigma_{x}(n)$ defined by (1.4) are dependent on $x \in \mathbb{T}$.

If the walk is symmetric (see (1.3)) and $\alpha$ is Diophantine, the fact that $\ln (1-\mathfrak{p})-\ln \mathfrak{p}$ is a smooth coboundary above $R_{\alpha}$ implies that the sums $\Sigma_{x}(n)$ are bounded, which renders the Diophantine walk very similar to the simple symmetric random walk (we will come back to this in $\$ 2.1$ and we refer the reader to $[6,8]$ for more details).

A contrario, obtaining various specific behaviors for the sums $\Sigma_{x}(n)$ of a generic function $\mathfrak{p} \in C^{\infty}(\mathbb{T},(0,1))$ when $\alpha$ is Liouville, underlies all our findings. Displaying very different behaviors of $\Sigma_{x}(n)$ at different time scales $n$ and different initial conditions $x$ is the key behind the erratic behavior of the Liouville walks.

Outline of the proofs. Section 3 contains the necessary preliminaries. To keep the exposition as clear as possible, we split the analysis of Liouville walks into two separate parts. In the first part (Sections 4-6) we deal with a fixed environment and describe several criteria based on the behavior of the potential, that imply various types of behaviors for the random walk.

In Section 4, we formulate criteria for localization, one-sided drift and two-sided drift for random walks in a fixed environment. The proofs are given in Section 6; they rely on auxillary estimates of exit times for random walks in a fixed environment presented in Section 5.

The second part of the paper (Sections 7-8) deals with quasi-periodic walks. In Section 7 we prove Theorem 7.1, stating that when $\alpha$ is Liouville, then for a residual set of symmetric environments, the criteria for localization, one-sided drift and two-sided drift are satisfied for almost every $x \in \mathbb{T}$. In fact, as we mentioned above, the criteria ask for particular behaviors of the sums $\Sigma_{x}(n)$ at different time scales $n$ and different initial conditions $x$. By definition of the criteria, it will be easy to show that the set of $\mathfrak{p} \in \mathcal{P}$ for which these criteria are satisfied contains a countable intersection of countable unions of open sets. These open sets, are subsets of $\mathfrak{p} \in \mathcal{P}$ for which a criterion on $\Sigma_{x}(n)$ holds for some $n$ and some (not too small) intervals of initial conditions $x$.

To prove the theorem, we just need to show that the union of these open sets is dense. For this we start by perturbing any given $\mathfrak{p} \in \mathcal{P}$ into a smooth multiplicative coboundary $\overline{\mathfrak{p}}$ above $R_{\alpha}$. Then, the main construction is to show that any smooth coboundary $\overline{\mathfrak{p}}$ can be perturbed to $\overline{\mathfrak{p}}+e_{n}(\cdot) \in \mathcal{P}$ that satisfies each of the above mentioned criteria at different scales. This is stated in the main Proposition 7.3. §7.1 contains the reduction of Theorem 7.1 to Proposition 7.3 while the rest of Section 7 is devoted to the proof of Proposition 
Random walks in a Liouville quasi-periodic environment

7.3. The proof proceeds by a Liouville construction in which we obtain $e_{n}$ and prove the required properties of the ergodic sums as in (1.4) for the function $\overline{\mathfrak{p}}+e_{n}$.

Section 8 contains the proofs of all the statements about Liouville walks, including the asymmetric walks that are treated in §8.6. We note that the proofs in $\S 8.6$ do not use the results of Sections 4-6 so the reader who is only interested in the asymmetric walks could skip those sections.

In the appendix we give the proof of the results in the generic deterministic elliptic medium. They are similar, albeit easier because we have more freedom in perturbing the medium, to the proofs in the Liouville environments.

\section{Results}

In this section we present our main results about Liouville walks and compare them with other classes of random walks. We start with a summary on what is known for Diophantine walks. Then we state our results on Liouville walks. After that we give a brief list of erratic behaviors of a walk in a generic deterministic elliptic medium. Next, we ask some natural questions that arise from our results. Finally, we end the section with a very brief survey of related results in the case of random walks in independent random media.

\subsection{Diophantine walks}

In this section we review the known results about quasi-periodic Diophantine walks. These results show that Diophantine walks are very similar to the simple random walks.

Recall the notations introduced in Definition 1.1. In particular, recall the notation $\mathcal{P}^{c}=C^{\infty}(\mathbb{T},(0,1)) \backslash \mathcal{P}$ where $\mathcal{P}$ is the set of functions satisfying the symmetry condition (1.3). The following results are known.

Theorem 2.1. ([25, Theorem 1]) (Stationary measure) For $\alpha \notin \mathbb{Q}$ and $\mathfrak{p} \in C^{\infty}(\mathbb{T},(0,1))$ such that any of the following two conditions holds:

(1) $\mathfrak{p} \in \mathcal{P}^{c}$,

(2) $\alpha$ is Diophantine,

then there exists a unique probability measure $\nu$ on $\mathbb{T}$ that is stationary for the process $\left(X_{t}\right)_{t \in \mathbb{N}}$ defined as in (1.2), and this measure is absolutely continuous with respect to the Haar measure on $\mathbb{T}$. Moreover, for each $\phi \in C^{0}(\mathbb{T}, \mathbb{R})$ and for any $x \in \mathbb{T}$

$$
\lim _{t \rightarrow \infty} \mathbb{E}_{x}\left(\phi\left(X_{t}\right)\right)=\nu(\phi) .
$$

The precise definition of a stationary measure will be given in §3.1.

For Diophantine frequencies, the walk $\left(Z_{t}\right)_{t \in \mathbb{N}}$ satisfies the Central Limit Theorem, as shown by the following two statements. Denote

$$
\Phi(z)=\int_{-\infty}^{z} \frac{1}{\sqrt{2 \pi}} e^{-u^{2} / 2} d u .
$$

Theorem 2.2. ([1], [25, Equations (15) and (20)]) For $\alpha$ Diophantine and $\mathfrak{p} \in C^{\infty}(\mathbb{T},(0,1))$, and the walk $\left(Z_{t}\right)_{t \in \mathbb{N}}$ defined as in (1.1), there exist $\mathbf{v} \in \mathbb{R}$ and $\sigma>0$ such that for all $x$

$$
\lim _{t \rightarrow \infty} \mathbb{P}_{x}\left(Z_{t}-t \mathbf{v}<\sigma \sqrt{t} z\right)=\Phi(z) .
$$

Therefore

$$
\lim _{t \rightarrow \infty} \mathbb{P}_{\mathrm{Leb}}\left(Z_{t}-t \mathbf{v}<\sigma \sqrt{t} z\right)=\Phi(z) .
$$

Moreover $\mathbf{v}=0$ iff the walk is symmetric. 
Note that Theorem 2.2 shows that the Diophantine walks behave similarly to simple random walks independently of the starting point on the circle. Namely, they have linear growth, variance also grows linearly and the limit distribution is Gaussian.

In fact, asymmetric walks $\left(Z_{t}\right)_{t \in \mathbb{N}}$ for all irrational frequencies, have quenched limits similar to simple random walks, but with a drift that depends on the starting point.

Theorem 2.3. ([6, Theorem B.2]) For $\alpha \notin \mathbb{Q}$ and $\mathfrak{p} \in \mathcal{P}^{c}$, and the walk $\left(Z_{t}\right)_{t \in \mathbb{N}}$ defined as in (1.1), there exist functions $\left(b_{t}(\cdot)\right)_{t \in \mathbb{Z}}$ and a number $\sigma>0$ such that for any $z \in \mathbb{R}$

$$
\lim _{t \rightarrow \infty} \mathbb{P}_{x}\left(Z_{t}-b_{t}(x)<\sigma \sqrt{t} z\right)=\Phi(z)
$$

In case $\alpha$ is Diophantine, we have that for every $x$, we can take $b_{t}(x)=t \mathbf{v}$ where $\mathbf{v}$ is given by Theorem 2.2.

Remark 2.4. A general formula for $b_{t}(\cdot)$ will be recalled in $\S 8.6$ (see equation (8.2)).

The results of Theorems 2.1-2.3 have been extended to random walks driven by rotations of $\mathbb{T}^{d}$, for arbitrary $d \in \mathbb{N}$, to random walks with bounded jumps where the walker can move from $x$ to $x+j \alpha$ with $|j| \leq L$ for some $L>1$ and to quasi-periodic walks on the strip, see $[3,6,7,8]$.

\subsection{Liouville walks}

Theorems 2.1, 2.2 and 2.3 naturally raise the question of what would be the behavior of a quasi-periodic walk when the driving frequency $\alpha$ is Liouville. The following statements show that their behavior can indeed be very different from the Diophantine case.

Recall that $\mathcal{P} \subset C^{\infty}(\mathbb{T},(0,1))$ the set of functions satisfying the symmetry condition (1.3), and $\Phi(z)$ is the normal distribution given by (2.1).

Theorem A. For any Liouville $\alpha$ there exists a dense $G_{\delta}$ set $\mathcal{R}=\mathcal{R}(\alpha) \subset \mathcal{P}$, and for each $\mathfrak{p} \in \mathcal{R}(\alpha)$ a set $S(\mathfrak{p}) \subset \mathbb{T}$ of full measure with the following property.

Let $\mathfrak{p} \in \mathcal{R}(\alpha)$ and $x \in S(\mathfrak{p})$, and consider a quasi-periodic walk $\left(Z_{t}\right)_{t \in \mathbb{N}}$ as in (1.1) in the quasi-periodic environment defined by $(\mathfrak{p}, \alpha, x)$. Then there exist strictly increasing sequences of integers $\left(r_{n}\right)_{n \in \mathbb{N}},\left(s_{n}\right)_{n \in \mathbb{N}},\left(t_{n}\right)_{n \in \mathbb{N}}$, and a sequence of positive integers $\left(\varepsilon_{n}\right)_{n \in \mathbb{N}}$ with $\varepsilon_{n} \rightarrow 0$, such that for any $\varepsilon>0$ and for $k$ sufficiently large we have:

(a) (Localization) For $T=r_{n}$, it holds

$$
\mathbb{P}_{x}\left(\max _{t \leq T}\left|Z_{t}\right|>16(\ln T)^{2}\right)<T^{-2} \text { and } \operatorname{Var}_{x}\left(Z_{T}\right)<300(\ln T)^{4}
$$

(b) (One-sided positive drift) For $T=s_{n}$ and some $\mu_{n}(x)>T^{1-\varepsilon}$, for any $z \in \mathbb{R}$, it holds

$$
\left|\mathbb{P}_{x}\left(\frac{Z_{T}-\mu_{n}(x)}{\sigma_{n}(x)}<z\right)-\Phi(z)\right|<\varepsilon, \quad\left|\frac{\ln \sigma_{n}(x)}{\ln T}-\frac{1}{2}\right|<\varepsilon
$$

where $\sigma_{n}(x)=\sqrt{\operatorname{Var}_{x}\left(Z_{T}\right)}$;

(c) (Two-sided drift) For $T=t_{n}$ there exist $b_{n}(x), b_{n}^{\prime}(x) \in\left[0.3 T^{1 / 5}, 0.4 T^{1 / 5}\right]$ and $\varepsilon_{n} \rightarrow 0$ such that

$$
\mathbb{P}_{x}\left(\left|Z_{T}-b_{n}(x)\right|<\varepsilon_{n} T^{1 / 5}\right)>0.1, \quad \mathbb{P}_{x}\left(\left|Z_{T}+b_{n}^{\prime}(x)\right|<\varepsilon_{n} T^{1 / 5}\right)>0.1
$$

Naturally, statement (b) can be modified to provide a one-sided negative drift for the walks over a subsequence of times.

We will also need a different version of property (b) in order to guarantee the absence of an annealed limit for the walk. Namely, we need a sequence of times so that property 
(b) holds with uniform drift parameters for a set of positive measure of initial positions $x \in \mathbb{T}$.

Theorem B. For any Liouville $\alpha$, there exists a dense $G_{\delta}$ set $\mathcal{R}^{\prime}=\mathcal{R}^{\prime}(\alpha) \subset \mathcal{P}$ with the following property.

(a) There exists a strictly increasing sequence of integers $\left(u_{n}\right)_{n \in \mathbb{N}}$, and sequences $\left(\sigma_{n}\right)_{n \in \mathbb{N}},\left(\varepsilon_{n}\right)_{n \in \mathbb{N}}$ with $\varepsilon_{n} \rightarrow 0$, such that for any $\mathfrak{p} \in \mathcal{R}^{\prime}(\alpha)$, for any $x \in \mathbb{T}$, the quasiperiodic random walk $\left(Z_{t}\right)_{t \in \mathbb{N}}$ as in (1.1) in the environment defined by $(\mathfrak{p}, \alpha, x)$ satisfies for each $T=u_{n}$ and any $z \in \mathbb{R}$ :

$$
\left|\mathbb{P}_{x}\left(Z_{T}<\sigma_{n} \sqrt{T} z\right)-\Phi(z)\right|<\varepsilon_{n} .
$$

(b) There exists a strictly increasing sequence of integers $\left(v_{n}\right)_{n \in \mathbb{N}}$ and two sequences of measurable subsets of $\mathbb{T},\left(\mathcal{I}_{n}\right)_{n \in \mathbb{N}},\left(\mathcal{I}^{\prime}{ }_{n}\right)_{n \in \mathbb{N}}$ with $\operatorname{Leb}\left(\mathcal{I}_{n}\right)>0.001$ and $\operatorname{Leb}\left(\mathcal{I}^{\prime}{ }_{n}\right)>0.001$, such that for any $\mathfrak{p} \in \mathcal{R}^{\prime}(\alpha)$, for any $x \in \mathcal{I}_{n} \cup \mathcal{I}^{\prime}{ }_{n}$ there exist $\mu_{n}(x)$ such that $\mu_{n}(x)>v_{n}^{1-\varepsilon_{n}}$ if $x \in \mathcal{I}_{n}$ and $\mu_{n}(x)<-v_{n}^{1-\varepsilon_{n}}$ if $x \in \mathcal{I}^{\prime}{ }_{n}$, and such that the following holds.

The quasi-periodic random walk $\left(Z_{t}\right)_{t \in \mathbb{N}}$ as in (1.1) in the environment defined by $(\mathfrak{p}, \alpha, x)$ satisfies for each $T=v_{n}$ and any $z \in \mathbb{R}$ :

$$
\left|\mathbb{P}_{x}\left(\frac{Z_{T}-\mu_{n}(x)}{\sigma_{n}(x)}<z\right)-\Phi(z)\right|<\varepsilon_{n}, \quad\left|\frac{\ln \sigma_{n}(x)}{\ln T}-\frac{1}{2}\right|<\varepsilon_{n},
$$

where $\sigma_{n}(x)=\sqrt{\operatorname{Var}_{x}\left(Z_{T}\right)}$.

Part (a) of Theorem B is based on the fact that for a generic $\mathfrak{p} \in \mathcal{P}$, the quasi-periodic walk $\left(Z_{t}\right)_{t \in \mathbb{N}}$ "simulates" (uniformly in $x \in \mathbb{T}$ ) a Diophantine walk for a sequence of times. The same phenomenon for asymmetric walks is observed in Theorem F. Theorem E encloses a similar observation, this time for every $\mathfrak{p} \in \mathcal{P}$ and for a generic set of $\alpha \in \mathbb{R}$.

As a byproduct of our analysis, we will show that the existence of an absolutely continuous stationary measure (see $\$ 3.1$ for the precise definition) is incompatible with the erratic behavior of Theorem A.

Corollary C. If $\alpha$ and $\mathfrak{p}$ are as in Theorem A, then the process $\left(X_{t}\right)_{t \in \mathbb{N}}$ defined by (1.2) has no absolutely continuous stationary measure on $\mathbb{T}$.

The proof of Corollary C will be given in §8.4. Its statement makes a sharp contrast with the behaviour for Diophantine walks, described in Proposition 3.1.

Theorems A and B imply that the walk does not satisfy any limit theorems. Let us give a more precise statement, with Definition 1.2 in mind.

Corollary D. If $\alpha$ and $\mathfrak{p}$ are as in Theorem A, then for $x$ in a set of full measure the walk $\left(Z_{t}\right)_{t \in \mathbb{N}}$ defined by (1.1) in the quasi-periodic environment defined by $(\mathfrak{p}, \alpha, x)$ has no quenched limit theorem at $x$.

If $\alpha$ and $\mathfrak{p}$ are as in Theorem B, then the walk $\left(Z_{t}\right)_{t \in \mathbb{N}}$ defined as in (1.1) has no annealed limit theorem.

Proof. We start with the absence of quenched limit theorems. Consider $x$ for which (b) and (c) of Theorem A hold. It follows from (b) that if a quenched limit theorem holds, then the limit distribution should be normal.

On the other hand, let $t_{n}$ be as in (c). Then, since $b_{n}(x)+b_{n}^{\prime}(x) \geq 0.6 t_{n}^{\frac{1}{5}}$, the normalization has to be at least of order $t_{n}^{\frac{1}{5}}$. Indeed, if the normalization was negligible compared to $t_{n}^{\frac{1}{5}}$ for infinitely many $n$, then the limit distribution would have to give weight larger than 0.1 to segments that accumulate at infinity, which is impossible. But if the normalization is comparable to $t_{n}^{\frac{1}{5}}$ or larger, then the limit distribution should give a probability larger than 0.1 to two intervals, each one having size at most $\varepsilon_{n}$. This implies 
Random walks in a Liouville quasi-periodic environment

that any limit point along the sequence $t_{n}$ has non-trivial atoms, so it cannot be normal, giving a contradiction.

To show that the walk has no annealed limit, we use Theorem B. From (a) it follows that if an annealed limit theorem holds, the limit must be the normal distribution.

On the other hand, (b) forces the normalization of any candidate annealed limit theorem to be of order at least $v_{n}^{1-\varepsilon}$ for the time $v_{n}$. Indeed, if the normalization was negligible compared to $v_{n}^{1-\varepsilon}$ for infinitely many $n$, then by (2.9) the limit distribution should give weight larger than 0.001 to segments that accumulate at infinity, which is impossible. But if the normalization is comparable to $v_{n}^{1-\varepsilon}$ or larger, then the CLT (2.9) would imply that the annealed limit distribution gives a positive mass to an interval of size $v_{n}^{-1 / 2+\varepsilon}$. This implies that any limit point along the sequence $v_{n}$ has non-trivial atoms, giving a contradiction with (a).

Our next result shows that for a generic Liouville frequency, the behavior of the corresponding walk "simulates" that of a Diophantine one (described by Theorem 2.2) for long periods of time.

Theorem E. There exists a dense $G_{\delta}$ set $\mathcal{A} \subset \mathbb{R}$ such that for any $(\alpha, \mathfrak{p}) \in \mathcal{A} \times \mathcal{P}$ there exist sequences $\left(\sigma_{n}\right)_{n \in \mathbb{N}}$ and $\left(T_{n}\right)_{n \in \mathbb{N}}, T_{n} \rightarrow \infty$, such that for any $x \in \mathbb{T}$ the quasi-periodic random walk $\left(Z_{t}\right)_{t \in \mathbb{N}}$ as in (1.1) in the environment defined by $(\alpha, \mathfrak{p}, x)$ satisfies for any $z \in \mathbb{R}$ :

$$
\left|\mathbb{P}_{x}\left(Z_{t}<\sigma_{n} \sqrt{t} z\right)-\Phi(z)\right|<\frac{1}{n} \text { for all } t \in\left[T_{n}, e^{T_{n}}\right],
$$

where $\Phi(z)$ is the normal distribution given by (2.1).

Consider $t$ of order $T_{n}$. Relation (2.10) implies that $\sigma_{n}$ is necessarily larger than $1 / \sqrt{T_{n}}$. Therefore, when $t$ is of order $e^{T_{n}}$, we see that the variance is almost linear in $t$.

Let us turn to the asymmetric quasi-periodic walks. Recall that, by Theorem 2.3, a quenched CLT (2.4) holds with some function $b_{t}(\cdot)$. Moreover, by Theorem 2.2, in the Diophantine case there exist $b_{t}(\cdot) \equiv t \mathbf{v}$ such that the annealed limit (2.3) holds. To show that no annealed limit theorem holds in the Liouville case, it suffices to show that the drift function $b_{t}$ in the quenched limit theorem fluctuates much more than $\sqrt{t}$ when we vary $x$.

Theorem F. For any Liouville $\alpha$ there exists a dense $G_{\delta}$ set $\mathcal{D}(\alpha) \subset \mathcal{P}^{c}$, such that the walk $\left(Z_{t}\right)_{t \in \mathbb{N}}$ defined by (1.1) with $\mathfrak{p} \in \mathcal{D}(\alpha)$, satisfies the following:

(a) There exists a sequence of integers $\left(s_{n}\right)_{n \in \mathbb{N}}$ and sequences $\left(b_{n}\right)_{n \in \mathbb{N}},\left(\sigma_{n}\right)_{n \in \mathbb{N}}$, $\left(\varepsilon_{n}\right)_{n \in \mathbb{N}}$ with $\varepsilon_{n} \rightarrow 0$, such that for $T=s_{n}$ we have for any $x \in \mathbb{T}$ and any $z \in \mathbb{R}$ :

$$
\left|\mathbb{P}_{x}\left(Z_{T}-b_{n}<\sigma_{n} \sqrt{t} z\right)-\Phi(z)\right|<\varepsilon_{n} .
$$

(b) There exists a sequence of integers $\left(t_{n}\right)_{n \in \mathbb{N}}$, and sequences $\left(\mathcal{J}_{n}\right)_{n \in \mathbb{N}}$ and $\left(\mathcal{J}_{n}^{\prime}\right)_{n \in \mathbb{N}}$ of measurable subsets of $\mathbb{T}$, such that the drift coefficients $b_{t_{n}}(\cdot)$ and $b_{s_{n}}(\cdot)$ of the quenched CLT (2.4) satisfy:

(i) $\mu\left(\mathcal{J}_{n}\right)>0.8$ and $\mu\left(\mathcal{J}_{n}^{\prime}\right)>0.1$;

(ii) For $x \in \mathcal{J}_{n}$ we have $\left|b_{t_{n}}(x)-b_{n}\right|<t_{n}{ }^{1 / 4}$, and for $x \in \mathcal{J}_{n}^{\prime}$ we have $b_{t_{n}}(x)>b_{n}+t_{n}{ }^{0.9}$.

As a consequence we get:

Corollary G. For $\alpha$ and $\mathfrak{p}$ as in Theorem $\mathrm{F}$, the walk $\left(Z_{t}\right)_{t \in \mathbb{N}}$ defined by (1.1) has no annealed limit. 
Random walks in a Liouville quasi-periodic environment

Proof. If an annealed limit as in (2.3) holds, then by (a) the limit must be the normal distribution. On the other hand, properties $(i)$ and $(i i)$ of $(b)$ require that the normalizing factor at time $t_{n}$ should satisfy $\sigma_{t_{n}}>t_{n}^{0.9}$. But then we get, again from $(i)$ and $(i i)$, that the limit distribution must give a mass larger than 0.8 to some point on the line, a contradiction with (a).

\subsection{Erratic behavior for random walks in generic deterministic elliptic envi- ronments}

In this section, we consider random walks on $\mathbb{Z}$ in a fixed generic deterministic elliptic environment $(\mathrm{p}(j))_{j \in \mathbb{Z}}$, that we define as follows.

Definition 2.5 (Deterministic elliptic environments and generic walks). For any $\varepsilon \in\left(0, \frac{1}{2}\right)$, we define $\mathcal{E}_{\varepsilon}=\{\mathrm{p}: \mathbb{Z} \rightarrow[\varepsilon, 1-\varepsilon]\}$. We call

$$
\mathcal{E}=\bigcup_{\varepsilon>0} \mathcal{E}_{\varepsilon}
$$

the set of deterministic elliptic environments. For every $\varepsilon>0$, we endow $\mathcal{E}_{\varepsilon}$ with the product topology generated by the sets of the form

$$
W_{\delta, K}(\overline{\mathrm{p}})=\{\mathrm{p}:|\mathrm{p}(n)-\overline{\mathrm{p}}(n)|<\delta \text { for }|n| \leq K\} .
$$

A subset $\overline{\mathcal{E}}$ of $\mathcal{E}$ is called generic if for each $\varepsilon, \overline{\mathcal{E}} \cap \mathcal{E}_{\varepsilon}$ contains a countable intersection of open dense sets.

For $\mathrm{p} \in \mathcal{E}$, we let $\left(\bar{Z}_{t}\right)_{t \in \mathbb{N}}$ be the random walk on $\mathbb{Z}$ given by $\bar{Z}_{0}=0$ and

$$
\mathbb{P}\left(\bar{Z}_{t+1}=k+1 \mid \bar{Z}_{t}=k\right)=\mathrm{p}_{k}, \quad \mathbb{P}\left(\bar{Z}_{t+1}=k-1 \mid \bar{Z}_{t}=k\right)=\mathrm{q}_{k} .
$$

We say that a property is satisfied by a generic random walk on $\mathbb{Z}$ if it is satisfied by the walks (2.13) for $\mathrm{p}$ in a generic subset of $\mathcal{E}$.

In the definition of generic sets of environments we are allowed to perturb any given environment outside of a finite set, so we can directly prescribe the asymptotic behavior of the potential to enforce a desirable behavior of the walk. Hence the erratic behavior similar to the Liouville walks can be observed for random walks in generic deterministic elliptic environments. The results are even stronger in the latter case and the proofs are much easier. Since we could not find these results in the literature, we give the proofs in the appendix.

The criteria that yield erratic behavior of generic walks that are included in the appendix are a source of inspiration for the criteria that we use in the Liouville walks context. However, the latter criteria must be more sophisticated and tailored in a way that makes it convenient to verify their validity for quasi-periodic Liouville walks.

Theorem 2.6. There exist a generic set $\overline{\mathcal{E}} \subset \mathcal{E}$ such that for each $\mathrm{p} \in \overline{\mathcal{E}}$, the following properties are satisfied by the walk $\left(\bar{Z}_{t}\right)_{t \in \mathbb{N}}$ defined in (2.13):

(a) (Recurrence) The walk is recurrent.

Moreover, there exist strictly increasing sequences $r_{k}, s_{k}, t_{k}$ such that

(b) (Localization) For $T=r_{k}$

$$
\mathbb{P}\left(\left|\bar{Z}_{T}\right|>(\ln T)^{2}\right)<T^{-1 / 2} \text { and } \operatorname{Var}\left(\bar{Z}_{T}\right)<2(\ln T)^{4} .
$$

(c) (One-sided drift) For $T=s_{n}$ and some $\mu_{n}, \sigma_{n}$ such that

$$
\liminf _{n \rightarrow \infty} \frac{\mu_{n}(x)}{s_{n}}>0, \quad \liminf _{n \rightarrow \infty} \frac{\sigma_{n}(x)}{\sqrt{s_{n}}}>0
$$

we have that for all $z$ 
Random walks in a Liouville quasi-periodic environment

$$
\mathbb{P}\left(\frac{\bar{Z}_{T}-\mu_{n}}{\sigma_{n}(x)}<z\right)=\Phi(z) .
$$

(d) (Two-sided drift) For $T=t_{n}$ there exists $b_{n}$ and $\varepsilon_{n}$ such that $\liminf _{n \rightarrow \infty} \frac{b_{n}}{t_{n}}>0$, $\lim _{n \rightarrow \infty} \varepsilon_{n}=0$ and

$$
\mathbb{P}\left(\left|\bar{Z}_{T}-b_{n}\right|<\varepsilon_{n} b_{n}\right)>0.1, \quad \mathbb{P}\left(\left|\bar{Z}_{T}+b_{n}\right|<\varepsilon_{n} b_{n}\right)>0.1 .
$$

As mentioned above, the proof of this theorem involves similar ideas, albeit simpler, as the ones used to prove the analogous results for Liouville walks. Therefore some readers may prefer to go through the proofs of Theorem 2.6 before going over the proofs of the results for the Liouville walks.

We note several interesting consequences of Theorem 2.6.

We say that the walk satisfies a limit theorem if there exist sequences $\left(b_{t}\right)_{t \in \mathbb{N}}$ and $\left(\sigma_{t}\right)_{t \in \mathbb{N}}$ and a proper (that is, not concentrated on a single point) distribution $\mathcal{D}(\cdot)$ such that for any $z \in \mathbb{R}$

$$
\lim _{t \rightarrow \infty} \mathbb{P}\left(\bar{Z}_{t}-b_{t}<\sigma_{t} z\right)=\mathcal{D}(z) .
$$

Corollary 2.7. There exist a generic set $\overline{\mathcal{E}} \subset \mathcal{E}$ such that for each $\mathrm{p} \in \overline{\mathcal{E}}$, the following properties are satisfied by the walk $\left(\bar{Z}_{t}\right)_{t \in \mathbb{N}}$ :

(a) $\liminf _{t \rightarrow \infty} \frac{\ln \left|\mathbb{E}\left(\bar{Z}_{t}\right)\right|}{\ln t}=0$, $\limsup _{t \rightarrow \infty} \frac{\ln \left|\mathbb{E}\left(\bar{Z}_{t}\right)\right|}{\ln t}=1$;

$$
\liminf _{t \rightarrow \infty} \frac{\ln \left(\operatorname{Var}\left(\bar{Z}_{t}\right)\right)}{\ln t}=0, \quad \limsup _{t \rightarrow \infty} \frac{\ln \left(\operatorname{Var}\left(\bar{Z}_{t}\right)\right)}{\ln t}=2 .
$$

(b) $\left(\bar{Z}_{t}\right)_{t \in \mathbb{N}}$ does not satisfy a limit theorem.

Proof. (a) Follows from parts (b) and (d) of Theorem 2.6.

Part (b) follows exactly as the absence of quenched limit in corollary D follows from Theorem A.

\subsection{Open questions}

We close Section 2 with two open questions about the Liouville one dimensional walks.

Comparing Theorem 2.1 with Corollary C leads to the following natural question.

Question 2.8. Suppose that the walk is symmetric and $\alpha$ is Liouville. By compactness, the walk has at least one stationary measure. Is the stationary measure unique? Are ergodic stationary measures mixing?

We also note that the maximal growth exponent for the variance of a generic walk obtained in Corollary 2.7(a) is optimal, since the variance of $Z_{T}$ is at least $\mathcal{O}(1)$ and at most $T^{2}$. However for Liouville walks we can only show that the variance grows along a subsequence at a rate that is not slower than $T^{1-\varepsilon}$, see the discussion after Theorem E. We believe that the optimal result should be of the same order as for the generic walks. Thus we formulate

Conjecture 2.9. For generic quasi-periodic symmetric walks, for almost every $x$

$$
\lim \sup _{T \rightarrow \infty} \frac{\ln \operatorname{Var}_{x}\left(Z_{T}\right)}{\ln T}=2 .
$$


Random walks in a Liouville quasi-periodic environment

\subsection{Random walks in independent random environments. A brief literature review}

A random environment is defined by a sequence $\left\{\mathrm{p}_{n}\right\}$ of random variables. We can generate it as $\mathrm{p}_{n}=\mathfrak{p}\left(\mathcal{T}^{n} \omega\right)$ where $\mathcal{T}$ is a map of a space $\Omega$ onto itself, and $\mathfrak{p}: \Omega \rightarrow[0,1]$ is a measurable function. It is usually assumed that $\mathcal{T}$ preserves a probability measure $\mu$. The most studied system in this class are iid environments where $\left\{\mathrm{p}_{n}\right\}$ are independent for different $n$. We consider a random walk in this environment where the walker moves to the right with probability $\mathrm{p}_{n}$ and to the left with probability $\mathrm{q}_{n}=1-\mathrm{p}_{n}$. The case where $\omega$ is distributed according to $\mu$ is called annealed, and the case where $\omega$ is fixed and we wish to obtain the results for $\mu$ almost every $\omega$ is called quenched. Quasi-periodic random walks are examples of random walks in random environment.

Since there is a vast literature on this subject, we will just briefly discuss the iid walks here, referring the readers to [27, Part I] for more information. Let $\Delta=\mathbb{E}\left(\ln \mathrm{p}_{n}-\ln \mathrm{q}_{n}\right)$. We call the walk symmetric if $\Delta=0$ and asymmetric otherwise. Let the walk start at the origin, and denote by $Z_{t}$ the position of the walker at time $t$. Here are some results.

According to [26], the walk is recurrent iff it is symmetric. Moreover, if $\Delta>0$, then $Z_{t} \rightarrow+\infty$ with probability 1 , and if $\Delta<0$ then $Z_{t} \rightarrow-\infty$ with probability 1 . Surprisingly, in the transient case the walk can escape to infinity with zero speed or have positive speed and superdiffusive fluctuations. We also note that, unless the walk satisfies the classical Central Limit Theorem, there is no quenched limits. ${ }^{1}$

Thus, the behavior of the random walk in the independent asymmetric environment can be very different from that of a simple random walk. The difference is even more startling in the symmetric case where it was shown by Sinai ([24]) that $\frac{Z_{t}}{\ln ^{2} t}$ converges to a non-trivial limit (the density of the limit distribution is obtained in [17]). The quenched distribution has even stronger localization properties, namely, most of the time the walker is localized on the scale $\mathcal{O}(1)$ [12]. More precisely, given $\varepsilon>0$, we can find an integer $N(\varepsilon)$ such that for each $n \in \mathbb{N}$, for a set of environments $\omega$ of measure more than $1-\varepsilon$, there is a subset $\mathfrak{T}_{t}(\omega) \subset \mathbb{Z}$ of cardinality $N(\varepsilon)$ such that $\mathbb{P}_{\omega}\left(Z_{t} \in \mathfrak{T}_{t}(\omega)\right)>1-\varepsilon$. This strong localization could be used to show that the symmetric walk does not satisfy a quenched limit theorem. The fact that the fluctuations of the walk in both annealed and quenched case are subpolynomial is referred to as Sinai-Golosov localization.

To understand different behaviors in different regimes in the asymmetric case, one needs a notion of a trap. Informally, a trap is a short segment $I$ where for most of the sites the drift is pointing in the direction opposite to the one in which the walker is going. The most convenient way to do this ([24]) is in terms of the potential, defined in (1.4). Namely, a segment $I$ is a trap if the minimal value of the potential inside $I$ is much smaller than both boundary values. The creation of traps is our main tool for proving the localization of the walker in the Liouville case.

\section{Preliminaries}

\subsection{Stationary measures}

Here we comment on the relation between our findings and the question of the existence of absolutely continuous stationary measures for the quasi-periodic walks. We consider a walk given by a pair $(\alpha, \mathfrak{p})$ with $\alpha \notin \mathbb{Q}$ and $\mathfrak{p} \in C^{\infty}(\mathbb{T},(0,1))$. The associated process $\left(X_{t}\right)_{t \in \mathbb{N}}$ has an absolutely continuous stationary measure with density $\rho(\cdot)$ iff for Haar a.e. $x \in \mathbb{T}$

$$
\rho(x)=\mathfrak{p}(x-\alpha) \rho(x-\alpha)+\mathfrak{q}(x+\alpha) \rho(x+\alpha) .
$$

\footnotetext{
${ }^{1}$ We refer the readers to $[19,22,5,10,21]$ for more discussion of the quenched behavior of the walk.
} 
A direct computation shows that the flux

$$
f(x)=\mathfrak{p}(x) \rho(x)-\mathfrak{q}(x+\alpha) \rho(x+\alpha)
$$

is constant along the orbit of the rotation and by ergodicity $f(x) \equiv f$. Now there are two cases

(I) The walk is not symmetric. We may assume (applying a reflection if necessary) that

$$
\int \ln \mathfrak{p}(x) d x>\int \ln \mathfrak{q}(x) d x .
$$

In this case we can take (rescaling $\rho$ if necessary) $f=1$ so that

$$
\rho(x)=\frac{1}{\mathfrak{p}(x)}+\frac{\mathfrak{q}(x+\alpha)}{\mathfrak{p}(x)} \rho(x+\alpha) .
$$

Iterating further one can obtain following [25] a smooth solution ${ }^{2}$

$$
\rho(x)=\frac{1}{\mathfrak{p}(x)} \sum_{k=0}^{\infty}\left(\prod_{j=1}^{k} \frac{\mathfrak{q}(x+j \alpha)}{\mathfrak{p}(x+j \alpha)}\right) .
$$

(II) The walk is symmetric. In this case using recursive analysis similar to case (I) one can see that there are no solutions with $f \neq 0$ (see [25]). In the case $f=0$ the equation reduces to

$$
\mathfrak{p}(x) \rho(x)=\mathfrak{q}(x+\alpha) \rho(x+\alpha) .
$$

Introducing

we see that (3.3) reduces to

$$
g(x)=\mathfrak{q}(x) \rho(x)
$$

$$
\frac{\mathfrak{q}(x)}{\mathfrak{p}(x)}=\frac{g(x)}{g(x+\alpha)} .
$$

We call the function $\mathfrak{p}(\cdot)$ such that (3.4) has a measurable solution $g$ a (multiplicative) coboundary above $\alpha$, and $g$ its corresponding transfer function ${ }^{3}$. In conclusion, we have the following proposition.

Proposition 3.1. (a) ([4, Theorem 3.1], [2, Theorem 1.8]). The Markov chain $\left(X_{t}\right)_{t \in \mathbb{N}}$ defined by $(\alpha, \mathfrak{p})$ as in (1.2) has a stationary measure which is absolutely continuous with respect to the Lebesgue measure iff either the walk is asymmetric or if it is symmetric and $\mathfrak{p}$ is a coboundary above $\alpha$.

(b) ([8, Corollary 6.2]) In the case $\alpha \in \mathbb{R} \backslash \mathbb{Q}$ and $\mathfrak{p}$ is a coboundary, the walk $\left(Z_{t}\right)_{t \in \mathbb{N}}$ defined by $(\alpha, \mathfrak{p})$ as in (1.1) satisfies the CLT. That is, there exists a constant $D^{2}>0$ such that for all $x$ all $z$

$$
\mathbb{P}_{x}\left(Z_{n} \leq z D \sqrt{n}\right)=\Phi(z)
$$

Observe that for asymmetric walks, the existence of an absolutely continuous stationary measure for $\left(X_{t}\right)_{t \in \mathbb{N}}$, or equivalently the existence of a measurable solution to (3.2), is behind the validity of the quenched central limit of Theorem 2.3, proved in [6].

We denote by $\mathcal{B}_{\alpha} \subset \mathcal{P}$ the subset of functions $\mathfrak{p}(\cdot)$ such that (3.4) has a smooth solution $g$. Such $\mathfrak{p}$ is called smooth (multiplicative) coboundary above $\alpha$.

For every $x \in \mathbb{T}$, denote $\Sigma_{x}(0)=0$,

$$
\Sigma_{x}(n)= \begin{cases}\sum_{j=1}^{n} \ln \mathfrak{q}(x+j \alpha)-\ln \mathfrak{p}(x+j \alpha), & n \geq 1, \\ \sum_{j=n+1}^{0} \ln \mathfrak{p}(x+j \alpha)-\ln \mathfrak{q}(x+j \alpha), & n \leq-1 .\end{cases}
$$

\footnotetext{
${ }^{2}$ The convergence of the sum defining the solution $\rho$ is guaranteed by the asymmetry condition and the fact that $\alpha \notin \mathbb{Q}$.

${ }^{3}$ A coboundary $\mathfrak{p}(\cdot)$ above $\alpha$ is necessarily symmetric.
} 
Random walks in a Liouville quasi-periodic environment

Notice that if $\mathfrak{p} \in \mathcal{B}_{\alpha}$, then $\Sigma_{x}(n)$ has an easy expression

$$
\Sigma_{x}(n)=\ln g(x+\alpha)-\ln g(x+(n+1) \alpha),
$$

where $g$ is as in (3.4). This behavior of $\Sigma_{x}(n)$, as we will see in $\S 3.2$, renders the walk very similar to the simple random walk.

In the symmetric case of Theorem 2.2, a crucial observation is that for $\alpha$ Diophantine, any smooth $\mathfrak{p} \in \mathcal{P}$ is a smooth coboundary above $\alpha$. As explained in the introduction, the fact that for a Liouville $\alpha$, the generic function $\mathfrak{p} \in \mathcal{P}$ displays very different behaviors of $\Sigma_{x}(n)$ at different time scales $n$ and different initial condition $x$ is the key behind the phenomena described in Theorems A, E, and F and Corollaries C, D and G.

\subsection{A fundamental martingale}

Until the end of this section we will work with random walks on $\mathbb{Z}$ in deterministic elliptic environments as in Definition 2.5, and study the dependence of the behavior of such a walk on the potential defined in (1.4). Notice that for a fixed triple $(\alpha, \mathfrak{p}, x)$, the quasi-periodic walk defined by (1.1) is equivalent to the walk in a deterministic environment defined by $\mathrm{p}(j)=\mathfrak{p}(x+j \alpha)$ for all $j \in \mathbb{Z}$.

Recall that we fix a small $\varepsilon_{0} \leq 0.1$ and let

$$
\mathcal{E}_{\varepsilon_{0}}=\left\{\mathrm{p}: \mathbb{Z} \rightarrow\left[\varepsilon_{0}, 1-\varepsilon_{0}\right]\right\} .
$$

For $\mathrm{p} \in \mathcal{E}_{\varepsilon_{0}}$, let $\mathrm{q}(\cdot)=1-\mathrm{p}(\cdot)$. Consider the nearest-neighbor random walk $\left(Z_{t}\right)_{t \in \mathbb{N}}$ on $\mathbb{Z}$ defined by the transition probabilities $Z(0)=0$,

$$
\mathbb{P}\left(Z_{t+1}=j+1 \mid Z_{t}=j\right)=\mathrm{p}(j), \quad \mathbb{P}\left(Z_{t+1}=j-1 \mid Z_{t}=j\right)=\mathrm{q}(j) .
$$

Denote $\Sigma(0)=0$ and

$$
\Sigma(n)= \begin{cases}\sum_{j=1}^{n} \ln \mathrm{q}(j)-\ln \mathrm{p}(j), & n \geq 1, \\ \sum_{j=n+1}^{0} \ln \mathrm{p}(j)-\ln \mathrm{q}(j), & n \leq-1 .\end{cases}
$$

For $j<k$, we use the notation

$$
\Sigma(j, k):=\Sigma(k)-\Sigma(j) .
$$

Denote $M(0)=0, M(1)=1$,

$$
M(n)= \begin{cases}1+\sum_{k=1}^{n-1} \prod_{j=1}^{k} \frac{\mathrm{q}(j)}{\mathrm{p}(j)}, & n \geq 2, \\ -\sum_{k=n+1}^{0} \prod_{j=k}^{0} \frac{\mathrm{p}(j)}{\mathrm{q}(j)}, & n \leq-1 .\end{cases}
$$

Notice that

$$
M(n)= \begin{cases}\sum_{j=0}^{n-1} e^{\Sigma(j)}, & n \geq 1, \\ -\sum_{j=n}^{-1} e^{\Sigma(j)}, & n \leq-1 .\end{cases}
$$

It is straightforward that $M\left(Z_{t}\right)$ is a martingale under $\mathbb{P}$. The optional stopping theorem for the martingales implies that if $Z_{t_{0}}=n$ with $a<n<b$, and if $\tau$ is the 
first time such that $Z_{t_{0}+\tau}$ the walk reaches either $a$ or $b$, then $M\left(Z_{\min \left(\left|t_{0}+t\right|,\left|t_{0}+\tau\right|\right)}\right)$ is a martingale under $\mathbb{P}$. In particular for any $a<n<b$

$$
\mathbb{P}\left(Z_{t} \text { reaches } b \text { before } a \mid Z_{0}=n\right)=\frac{M(n)-M(a)}{M(b)-M(a)} .
$$

(see e.g. [9, Theorem 6.4.6]).

This formula provides a relation between the sums $\Sigma(j, k)$ and the behavior of the walk.

We note that (3.10) also holds if $a=-\infty$ or $b=+\infty$ (see e.g. [23, §VII.3]). In particular,

$$
Z_{t} \text { is recurrent } \Leftrightarrow \lim _{n \rightarrow-\infty} M(n)=-\infty \text { and } \lim _{n \rightarrow+\infty} M(n)=+\infty,
$$

and

$$
\mathbb{P}\left(\lim _{t \rightarrow+\infty} Z_{t}=+\infty\right)=\frac{M_{-}}{M_{-}+M_{+}} \text {where } M_{ \pm}=\lim _{n \rightarrow \pm \infty}|M(n)|
$$

\section{Random walks in a deterministic aperiodic medium. Diffusion and localization via optional stopping}

For a fixed $\varepsilon_{0} \leq 0.1$ and $\mathcal{E}$ as in (2.12), fix $\mathrm{p} \in \mathcal{E}$ and consider the random walk $\left(Z_{t}\right)_{t \in \mathbb{N}}$ defined by (3.7).

In this section we present criteria involving the sums $\Sigma(n)$ defined in (3.8), that will be used to guarantee the different behaviors in Theorem A.

Proposition 4.1 below gives a criterion for localization, Proposition 4.2 for one-sided drift, and Proposition 4.3 for two-sided drift. The proofs of these propositions will be given in Section 6.

\subsection{Localization criterion}

We say that p satisfies condition $\mathcal{C}_{1}(N)$ if the following two inequalities hold:

$$
\begin{aligned}
\Sigma(N) & >N^{1 / 2}, & & \left(\mathcal{C}_{1} a_{+}\right) \\
\Sigma(-N) & >N^{1 / 2} . & & \left(\mathcal{C}_{1} a_{-}\right)
\end{aligned}
$$

Proposition 4.1. If p satisfies condition $\mathcal{C}_{1}(N)$, then for $T=e^{\sqrt{N} / 4}$ we have

$$
\mathbb{P}\left(\max _{t \leq T}\left|Z_{T}\right|>16(\ln T)^{2}\right)<T^{-2} \text { and } \operatorname{Var}\left(Z_{T}\right)<300(\ln T)^{4}
$$

(Localization)

Condition $\left(\mathcal{C}_{1}\right)$ means that the origin is a sharp local minimum of the potential, and as explained in the introduction, it implies that the walker spends a lot of time near the origin.

\subsection{One-sided drift criterion}

We say that p satisfies condition $\mathcal{C}_{2}(N, \varepsilon)$ for $\varepsilon>0$ if there exist $A>100$ and $L$ satisfying $e^{e^{A}}<L \leq N^{\varepsilon^{2}}, N \leq e^{L^{0.1}}$, such that the following conditions hold:

$$
\begin{array}{ll}
\Sigma(-L)>\sqrt{L} & \left(\mathcal{C}_{2} a\right) \\
\Sigma\left(k, k^{\prime}\right)<A \text { for all } k, k^{\prime} \in[-N, N], k \leq k^{\prime} ; & \left(\mathcal{C}_{2} b\right) \\
|\mathrm{p}(j+k L)-\mathrm{p}(j)|<N^{-1 / \varepsilon^{3}} \text { for all }(j, k) \in[0, L-1] \times[-N / L, N / L] . & \left(\mathcal{C}_{2} c\right)
\end{array}
$$


Proposition 4.2. If p satisfies condition $\mathcal{C}_{2}(N, \varepsilon)$ for some $\varepsilon>0$, then for $T=N$, there exist $\mu>T^{1-\varepsilon}$ and $\sigma$ with $\left|\frac{\ln \sigma}{\ln T}-\frac{1}{2}\right|<\varepsilon$ such that for every $z \in \mathbb{R}$

$$
\left|\mathbb{P}\left(\frac{Z_{T}-\mu}{\sigma}<z\right)-\Phi(z)\right|<\varepsilon .
$$

(One-sided drift)

Conditions $\left(\mathcal{C}_{2}(a)\right)$ and $\left(\mathcal{C}_{2}(c)\right)$ imply that on a large segment around the origin, the potential $\Sigma$ is decreasing on scale $L$, while $\left(\mathcal{C}_{2}(b)\right)$ means that there are no deep wells of the potential (also known as traps) on smaller scales. Thus, Proposition 4.2 confirms the heuristic arguments described in the introduction after (1.4).

\subsection{Two-sided drift criterion}

We say that p satisfies condition $\mathcal{C}_{3}(N, \varepsilon)$ for $\varepsilon>0$ if there exist $A>100, e^{e^{A}}<$ $Q<N^{1 / 2}$ and numbers $u, v, w_{ \pm}, u^{\prime}, v^{\prime}, w_{ \pm}^{\prime}$ such that $v, v^{\prime} \in[0.3,0.4]$,

$$
\begin{array}{r}
0.225<u<v-\varepsilon<w_{-}<v<w_{+}<v+\varepsilon<0.5, \\
0.225<u^{\prime}<v^{\prime}-\varepsilon<w_{-}^{\prime}<v^{\prime}<w_{+}^{\prime}<v^{\prime}+\varepsilon<0.5,
\end{array}
$$

and

$$
\begin{aligned}
& \Sigma\left(v N, w_{+} N\right)>N^{1 / 2}, \quad \Sigma\left(v N, w_{-} N\right)>N^{1 / 2}, \\
& \Sigma\left(-v^{\prime} N,-w_{-}^{\prime} N\right)>N^{1 / 2}, \quad \Sigma\left(-v^{\prime} N,-w_{+}^{\prime} N\right)>N^{1 / 2}, \\
& \Sigma\left(k, k^{\prime}\right)<A \text { for } k, k^{\prime} \in\left[-u^{\prime} N, v N\right], \quad k^{\prime} \geq k \\
& \Sigma\left(k, k^{\prime}\right)<A \text { for } k, k^{\prime} \in\left[-v^{\prime} N, u N\right], \quad k^{\prime} \leq k \\
& \Sigma(k)=\Sigma(k)+B(k), \quad k \in\left[-v^{\prime} N, v N\right],
\end{aligned}
$$

where $\bar{\Sigma}$ and $B$ satisfy

$$
|\bar{\Sigma}(k)-\bar{\Sigma}(k+l Q)|<Q^{-1 / 2} \text { for } k \in[0, Q], l \in\left[-v^{\prime} N / Q, v N / Q\right]
$$

and

$$
B(k) \begin{cases}=0 & \text { for } k \in\left[-u^{\prime} N, u N\right] \\ \leq 0 & \text { for } k \in\left[-v^{\prime} N, v N\right]\end{cases}
$$

Figure 1 illustrates the behavior of $\Sigma(k)$ for $k \in\left(-\left(v^{\prime}+\varepsilon\right) N,(v+\varepsilon) N\right)$ due to $\left(\mathcal{C}_{3}\right)$. In the figure we assumed $\bar{\Sigma} \equiv 0$ since (4.1) and (4.2) imply that the effect of $\bar{\Sigma}$ is not important in the behavior of $\Sigma(k)$.

We note that condition $\left(\mathcal{C}_{3} a\right)$ implies localization of the walk around the points $-v^{\prime} N$ and $v N$ (compare with conditions of Proposition 4.1).

Conditions $\left(\mathcal{C}_{3} b_{ \pm}\right)$imply that the random walk starting at zero exits the interval $\left[-v^{\prime} N, v N\right]$ before time $N^{5}$ with probability almost one (see Lemma 5.2 below).

Condition $\left(\mathcal{C}_{3} c\right)$ compares the walk on $\left[-v^{\prime} N, v N\right]$ to a $Q$-periodic walk. This condition, combined with $\left(\mathcal{C}_{3} b_{ \pm}\right)$, makes sure that for the random walk starting at zero, both the probability of reaching $-v^{\prime} N$ before time $N^{5}$, and the probability of reaching $v N$ before time $N^{5}$, are not too small. Since $\left(\mathcal{C}_{3} a\right)$ implies localization around $-v^{\prime} N$ and $v N$ we get the following.

Proposition 4.3. If $\mathrm{p} \in \mathcal{C}_{3}(N, \varepsilon)$, then for any $T \in\left[N^{5}, e^{N^{1 / 4}}\right]$ we have:

$$
\left\{\begin{array}{l}
\mathbb{P}\left(Z_{T} \in\left[w_{-} N, w_{+} N\right]\right)>0.1 \\
\mathbb{P}\left(Z_{T} \in\left[-w_{+}^{\prime} N,-w_{-}^{\prime} N\right]\right)>0.1
\end{array}\right.
$$

(Two-sided drift) 
Random walks in a Liouville quasi-periodic environment

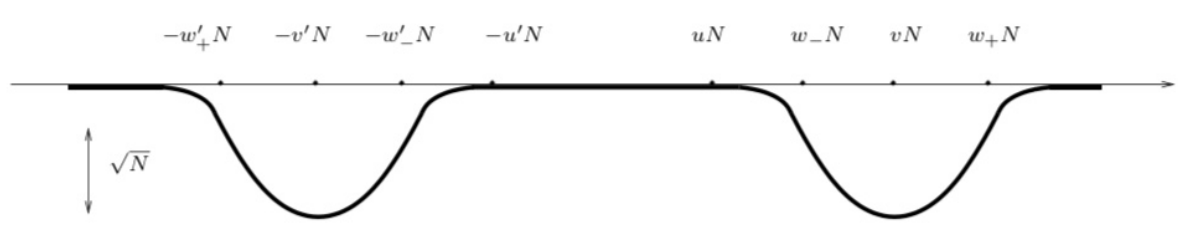

Figure 1: A sketch of the graph of $\Sigma(k)$ under condition $\mathcal{C}_{3}(N, \varepsilon)$, in which it is assumed that $\bar{\Sigma} \equiv 0$.

\section{Exit time estimates}

In this section we derive the key estimates used in the proof of the above propositions for $\mathrm{p} \in \mathcal{E}$, see (2.12). Recall the definitions of $\Sigma(n)$ and $M(n)$ given in (3.8) and (3.9).

\subsection{Traps}

Lemma 5.1. Suppose that for some $N$ condition $\left(\mathcal{C} a_{+}\right)$holds, i.e.,

$$
\Sigma(N)>\sqrt{N} .
$$

Then for $T=e^{\sqrt{N} / 2}$ we have

$$
\mathbb{P}\left(\max _{t \leq T} Z_{t}>N\right)<\exp (-\sqrt{N} / 2) .
$$

In the same way, if

$$
\Sigma(-N)>\sqrt{N}
$$

then for $T=e^{\sqrt{N} / 2}$ we have

$$
\mathbb{P}\left(\max _{t \leq T} Z_{t}<-N\right)<\exp (-\sqrt{N} / 2) .
$$

Moreover, if for some $N$ both $\left(\mathcal{C} a_{+}\right)$and $\left(\mathcal{C} a_{-}\right)$hold, then for $T_{1}=e^{\sqrt{N} / 4}$ we have:

$$
\operatorname{Var}\left(Z_{T_{1}}\right)<300\left(\ln T_{1}\right)^{4} \text {. }
$$

Proof. Recall the notations and the background material from $§ 3.2$. If $\left(\mathcal{C} a_{+}\right)$holds, then we have $M(N) \geq e^{\sqrt{N}}$, and (3.10) implies that

$$
\begin{aligned}
\mathbb{P}\left(Z_{t} \text { visits } N \text { before } 0 \mid Z_{0}=1\right) & =\frac{M(1)-M(0)}{M(N)-M(0)}=\frac{1}{M(N)} \\
& \leq \exp (-\sqrt{N}) .
\end{aligned}
$$

Hence, for $L=o\left(e^{\sqrt{N}}\right)$ we have:

$\mathbb{P}\left(Z_{t}\right.$ visits $N$ before visiting $0 L$ times $) \leq 1-\left(1-e^{-\sqrt{N}}\right)^{L} \leq 2 L \exp (-\sqrt{N})$.

Choosing $L=\exp (\sqrt{N} / 2)$, we obtain:

$$
\mathbb{P}\left(\max _{t \leq \exp (\sqrt{N} / 2)} Z_{t}>N\right)<2 \exp (-\sqrt{N} / 2) .
$$

The case of $\left(\mathcal{C} a_{-}\right)$is exactly similar.

Assume now that both $\left(\mathcal{C} a_{+}\right)$and $\left(\mathcal{C} a_{-}\right)$hold. Then for $T_{1}=\exp (\sqrt{N} / 4)$ we have $N=16\left(\ln T_{1}\right)^{2}$, and $\operatorname{Var}\left(Z_{T_{1}}\right) \leq \mathbb{E}\left(Z_{T_{1}}^{2}\right) \leq N^{2}+2 T_{1}^{2} \exp (-\sqrt{N} / 2)<N^{2}+2<300\left(\ln T_{1}\right)^{4}$. 


\subsection{Exit time in the absence of traps}

Let $L \in \mathbb{N}$. For an arbitrary choice of $k_{0} \in(-L, L)$, let $\tau$ be the first time the walk that starts at $k_{0}$ hits $L$ or $-L$.

Lemma 5.2. Suppose that there exist $A>100$ and $L$ satisfying $e^{e^{A}}<L$ such that for each $k \in[-L, L]$ either $\left(\mathcal{C} b_{+}\right)$or $\left(\mathcal{C} b_{-}\right)$holds:

$$
\begin{array}{cc}
\Sigma\left(k, k^{\prime}\right)<A \text { for all } k^{\prime} \in[-L, L], & k^{\prime} \geq k ; . \\
\Sigma\left(k, k^{\prime}\right)<A \text { for all } k^{\prime} \in[-L, L], & k^{\prime} \leq k .
\end{array}
$$

Then there is a constant $c>0$ such that for $s \in\{1,2,3\}$ we have

$$
\mathbb{E}\left(\tau^{s}\right) \leq c e^{s A} L^{2 s+1} \text {. }
$$

Moreover $\mathbb{E}(\tau) \geq L$ and $\operatorname{Var}(\tau) \geq 1$

Proof. For every $k \in I=[-L+1, L-1]$, let $\eta_{k}$ be the total time the walker (starting at 0 ) spends at site $k$ before reaching $-L$ or $L$. Then $\tau=\sum_{k \in I} \eta_{k}$. Hence, for any $s \in \mathbb{N}$

$$
\tau^{s} \leq L^{s} \sum_{k \in I} \eta_{k}^{s}
$$

Thus, it suffices to show that for $s \in\{1,2,3\}$ and for any $k \in I$

$$
\mathbb{E}\left(\eta_{k}^{s}\right) \leq c e^{s A} L^{s} \text {. }
$$

For $k \in I$, let $\bar{\eta}_{k}$ be the total time a walker starting at site $k$ spends at site $k$ before reaching $-L$ or $L$.

Note that $\bar{\eta}_{k}$ has geometric distribution with parameter

$$
r_{k}=\mathbb{P}(Z \text { starting at } k \text { does not return to } k \text { before exiting } I) \text {. }
$$

Since $\mathbb{E}\left(\eta_{k}^{s}\right) \leq \mathbb{E}\left(\bar{\eta}_{k}^{s}\right)$, we finish the proof of (5.1) once we prove the following

Claim. If either $\left(\mathcal{C} b_{+}\right)$or $\left(\mathcal{C} b_{-}\right)$holds, we have for every $k \in I$

$$
r_{k} \geq \frac{c}{L e^{A}}
$$

Proof of the Claim. Fix $k \in[-L, L]$. Observe that since the walk is elliptic (p $\in \mathcal{E})$, then there exists $c>0$ such that

$$
\begin{aligned}
r_{k} \geq \varepsilon_{0} \max & \left\{\mathrm{P}\left(Z \text { visits } L \text { before } k \mid Z_{0}=k+1\right),\right. \\
& \left.\mathbb{P}\left(Z \text { visits }(-L) \text { before } k \mid Z_{0}=k-1\right)\right\} .
\end{aligned}
$$

Now, if $\left(\mathcal{C} b_{+}\right)$holds, then (3.10) implies

$$
\begin{aligned}
\mathbb{P}\left(Z \text { visits } L \text { before } k \mid Z_{0}=k+1\right) & =\frac{M(k+1)-M(k)}{M(L)-M(k)}=\frac{e^{\Sigma(k+1)}}{\sum_{j=k+1}^{L-1} e^{\Sigma(j)}} \\
& =\frac{1}{1+\sum_{j=k+2}^{L-1} e^{\Sigma(k+1, j)}}>\frac{1}{L e^{A}} .
\end{aligned}
$$

In the same way, if $\left(\mathcal{C} b_{+}\right)$holds, then

$$
\mathrm{P}\left(Z \text { visits }(-L) \text { before } k \mid Z_{0}=k-1\right)>\frac{1}{L e^{A}},
$$

and the claim is proved.

Since the walker moves one step at a time, $\mathbb{E}(\tau) \geq L$. The lower bound on the variance of $\tau$ is obvious due to the ellipticity condition on the walk. 


\section{Proofs of the criteria}

In this section, we prove Propositions 4.1-4.3.

Proposition 4.1 follows directly from Lemma 5.1.

Proof of Proposition 4.2. Fix $N \in \mathbb{N}$ and $\varepsilon>0$ and $A>100$ and $L$ satisfying $e^{e^{A}}<L \leq$ $N^{\varepsilon^{2}}$, and $N \leq e^{L^{0.1}}$.

To make the argument easier to follow, we first consider the periodic case, i.e., we assume that the environment satisfies

$$
\mathrm{p}(k+L)=\mathrm{p}(k) \text { for any } k \in \mathbb{Z} .
$$

If we run the walk starting from 0 and stop it at the time $\tau$ when it hits either $L$ or $-L$, we get two random variables: $\tau$ and $U=Z_{\tau}$ (thus, $U$ takes values $L$ or $-L$ ). Let us consider iid copies $\left(\tau_{i}, U_{i}\right)$ of such pairs. Denote

$$
\hat{\mu}=\mathbb{E}\left(\tau_{i}\right), \quad \hat{V}=\mathbb{E}\left(\left(\tau_{i}-\hat{\mu}\right)^{2}\right), \quad \hat{\gamma}=\mathbb{E}\left(\left|\tau_{i}-\hat{\mu}\right|^{3}\right) .
$$

By Lemma 5.2 we have the following estimates:

$$
L \leq \hat{\mu} \leq c e^{A} L^{3}, \quad 1 \leq \hat{V} \leq c e^{2 A} L^{5}, \quad \hat{\gamma} \leq c e^{3 A} L^{7} .
$$

Note that

$$
\mathbb{P}(U=L) \geq 1-e^{-0.5 \sqrt{L}}
$$

by condition $\left(\mathcal{C}_{2} a\right)$ (cf. the proof of Lemma 5.1).

For $M \in \mathbb{N}$, denote

$$
\Theta_{M}:=\sum_{i=1}^{M} \tau_{i}
$$

For $M \leq N^{2}$ we have that $Z_{\Theta_{M}}=\sum_{i=1}^{M} U_{i}$ satisfies

$$
\mathbb{P}\left(Z_{\Theta_{M}}=M L\right) \geq\left(1-e^{-0.5 \sqrt{L}}\right)^{N^{2}} \geq 1-e^{-0.1 \sqrt{L}},
$$

if $N$ is sufficiently large, since $N \leq e^{L^{0.1}}$.

Define the stopping time $M_{N}$ as the first integer such that $\Theta_{M_{N}} \geq N$. By [14, Theorem 1], we have that the "residual lifetime" or "excess over the boundary", $\Theta_{M_{N}}-N$ satisfies

$$
\mathbb{E}\left(\Theta_{M_{N}}-N\right) \leq \hat{V} / \hat{\mu} \leq c e^{2 A} L^{4},
$$

from (6.2). Since $L>e^{e^{A}}$ Markov inequality implies that

$$
\mathbb{P}\left(\Theta_{M_{N}} \in\left[N, N+L^{6}\right]\right) \geq 1-\frac{1}{L} .
$$

Thus, combining (6.3) and (6.4), we get

$$
\mathbb{P}\left(\left|Z_{N}-M_{N} L\right| \leq 2 L^{6}\right) \geq 1-\frac{2}{L} .
$$

By the Berry-Esseen theorem for renewal counting processes [13, Theorem 2.7.1] we have

$$
\left|\mathbb{P}\left(\frac{M_{N}-\frac{N}{\hat{\mu}}}{\sqrt{\frac{\hat{V}}{\hat{\mu}^{3}}}}<z\right)-\Phi(z)\right|<4\left(\frac{\hat{\gamma}}{\sqrt{\hat{V}}}\right)^{3} \sqrt{\frac{\hat{\mu}}{N}}<\frac{1}{L}
$$


if $N$ (and therefore $L$ since $N \leq e^{L^{0.1}}$ ) is sufficiently large. Hence

$$
\left|\mathbb{P}\left(\frac{Z_{N}-\frac{L N}{\hat{\mu}}}{L \sqrt{\frac{\hat{V}}{\hat{\mu}^{3}} N}}<z\right)-\Phi(z)\right|<\frac{1}{\sqrt{L}} .
$$

Since $L \leq N^{\varepsilon^{2}}$, (6.2) implies that

$$
\mu:=\frac{L N}{\hat{\mu}}>\frac{N}{c e^{A} L^{2}}>N^{1-\varepsilon}
$$

and

$$
\sigma:=L \sqrt{\frac{\hat{V}}{\hat{\mu}^{3}} N}
$$

satisfies $|\ln \sigma / \ln N-1 / 2|<\varepsilon$.

This completes the proof in the periodic case (6.1). Now let the periodicity assumption (6.1) be replaced by the weaker condition $\left(\mathcal{C}_{2} c\right)$. In this case we consider a new periodic environment $\overline{\mathrm{p}}_{n}$, where $\overline{\mathrm{p}}_{n}=\mathrm{p}_{n}$ for each $n \in[0, L]$, and $\overline{\mathrm{p}}_{n}$ is periodic with period $L$. Let $\overline{\mathbb{P}}$ denote the corresponding probability for the paths of the walk $\left(\bar{Z}_{t}\right)_{t \in \mathbb{N}}$ defined by $\bar{p}$. By $\left(\mathcal{C}_{2} c\right)$, the conditions $\left(\mathcal{C}_{2} a\right)$ and $\left(\mathcal{C}_{2} b\right)$ are valid for $\left(\bar{Z}_{t}\right)_{t \in \mathbb{N}}$, thus the CLT limit (6.7) holds for $\bar{Z}_{N}$, with the corresponding $\overline{\hat{\mu}}, \overline{\hat{V}}$.

Now, for any path of length $N$ for the walks: $\left(z_{1}, \ldots, z_{N}\right), z_{j+1}=z_{j} \pm 1$, we have from $\left(\mathcal{C}_{2} c\right)$ that

$$
\left|\mathbb{P}\left(Z_{1}=z_{1}, \ldots, Z_{N}=z_{N}\right)-\overline{\mathbb{P}}\left(\bar{Z}_{1}=z_{1}, \ldots, \bar{Z}_{N}=z_{N}\right)\right|<N^{-1 / \varepsilon^{2}} \mathbb{P}\left(\bar{Z}_{1}=z_{1}, \ldots, \bar{Z}_{N}=z_{N}\right) .
$$

This shows that $|\overline{\hat{\mu}}-\hat{\mu}|<N^{-1}$ and $|\overline{\hat{V}}-\hat{V}|<N^{-1}$, and also that one can replace $\bar{Z}_{N}$ by $Z_{N}$ in (6.7). Hence, the general case follows from the periodic one.

Proof of Proposition 4.3. We divide the proof into three steps.

Step 1. For an arbitrary choice of $k \in\left(-v^{\prime} N, v N\right)$, denote by $\tau$ the exit time from $\left(-v^{\prime} N, v N\right)$ (while starting at $k$ ). We need to estimate

$$
\mathbb{P}\left(Z_{t} \text { reaches }-v^{\prime} N \text { or } v N \text { before time } N^{5} \mid Z_{0}=k\right)=\mathbb{P}\left(\tau<N^{5}\right) .
$$

By Lemma 5.2, under condition $\left(\mathcal{C}_{3} b\right)$ there exists $c>0$ such that for any $k \in$ $\left(-v^{\prime} N, v N\right)$ we have: $\mathbb{E}(\tau)<c e^{A} N^{3}$. Then $\mathbb{P}\left(\tau>N^{4}\right) N^{4}<\mathbb{E}(\tau)<c e^{A} N^{3}$, so

$$
\mathbb{P}\left(\tau>N^{4}\right)<c e^{A} / N .
$$

This implies

$$
\mathbb{P}\left(\tau>N^{5}\right)<\left(c e^{A} / N\right)^{N}<e^{-N}
$$

Hence,

$$
\mathbb{P}\left(Z_{t} \text { reaches }-v^{\prime} N \text { or } v N \text { before time } N^{5}\right)>1-e^{-N} .
$$

Step 2. We have the following two inequalities:

$$
\begin{aligned}
& \mathrm{P}\left(Z_{t} \text { visits }-v^{\prime} N \text { before visiting } v N\right) \leq 0.89 \\
& \mathrm{P}\left(Z_{t} \text { visits } v N \text { before visiting }-v^{\prime} N\right) \leq 0.89
\end{aligned}
$$

We prove the first estimate, the second one can be proved similarly. By (3.10)

$$
\mathbb{P}\left(Z \text { visits }\left(-v^{\prime} N\right) \text { before visiting } v N\right)=\frac{M(v N)}{M(v N)-M\left(-v^{\prime} N\right)} .
$$


Using $\left(\mathcal{C}_{3} c\right)$, we get:

$M(v N)=\sum_{j=1}^{v N} e^{\Sigma(j)} \leq \sum_{j=1}^{v N} e^{\bar{\Sigma}(j)} \leq \sum_{l=1}^{v N / Q+1} \sum_{j=1}^{Q} e^{\bar{\Sigma}(j+(l-1) Q)} \leq\left(\frac{v N}{Q}+1\right) M(Q)\left(1+2 Q^{-1 / 2}\right)$.

In the same way,

$$
M(u N)=\sum_{j=1}^{u N} e^{\Sigma(j)}=\sum_{j=1}^{u N} e^{\bar{\Sigma}(j)} \geq \sum_{l=1}^{u N / Q} \sum_{j=1}^{Q} e^{\bar{\Sigma}(j+(l-1) Q)} \geq \frac{u N}{Q} M(Q)\left(1-2 Q^{-1 / 2}\right) .
$$

Hence

Similarly

$$
M(v N) \geq M(u N) \geq \frac{u N}{Q} M(Q)\left(1-2 Q^{-1 / 2}\right) .
$$

$$
M\left(-v^{\prime} N\right) \leq M\left(-u^{\prime} N\right) \leq-\left(\frac{u^{\prime} N}{Q}-1\right) M(Q)\left(1-2 Q^{-1 / 2}\right)
$$

Hence,

$$
\frac{M(v N)-M(x)}{M(v N)-M\left(-v^{\prime} N\right)}<\frac{v}{u+u^{\prime}}+0.01<\frac{0.4}{0.45}+0.01<0.89 .
$$

Step 3. By Step 1, with probability $1-e^{-N}$, the walk starting at 0 reaches either $v N$ or $-v N^{\prime}$ before time $N^{5}$. By Step 2, it reaches $v N$ before time $N^{5}$ with probability larger than 0.1. The first part of $\left(\mathcal{C}_{3} a\right)$ states that $\Sigma\left(v N, w_{+} N\right)>N^{1 / 2}$ and $\Sigma\left(v N, w_{-} N\right)>N^{1 / 2}$. Under this condition, Lemma 5.1 implies that the walk starting at $v N$ satisfies

$$
\mathbb{P}\left(Z_{T} \in\left[w_{-} N, w_{+} N\right]\right)>0.1
$$

for all $T \in\left[N^{5}, e^{N^{1 / 4}}\right]$, which implies the desired result. The same argument holds for $-v^{\prime} N$.

\section{Quasi-periodic environments}

In this section we return to the study of quasi-periodic random walks. Fix a Liouville number $\alpha \in \mathbb{R} \backslash \mathbb{Q}$ and $\mathfrak{p} \in \mathcal{P}$, and for $x \in \mathbb{T}$ consider the corresponding environment:

$$
\mathrm{p}(j):=\mathfrak{p}(x+j \alpha),
$$

as well as the random walk $\left(Z_{t}\right)_{t \in \mathbb{N}}$ on it, defined by (1.1).

It will be convenient to reformulate the main conditions $\mathcal{C}_{j}, j=1,2,3$, in this new context. First of all, notice that condition $\mathcal{C}_{1}=\mathcal{C}_{1}(N)$ does not depend on $\varepsilon$, while the other two conditions do. For the uniformity of notations, we formally include an $\varepsilon$ in all the three conditions. We say that

$$
x \in \mathcal{C}_{j}(\mathfrak{p}, N, \varepsilon) \text { if and only if } \mathrm{p} \in \mathcal{C}_{j}(N, \varepsilon), \quad j=1,2,3 .
$$

The goal of this section is to prove the following statement.

Theorem 7.1. For any Liouville $\alpha$ there exists a dense $G_{\delta}$ set $\mathcal{R} \subset \mathcal{P}$ with the following property: for any $\mathfrak{p} \in \mathcal{R}$, for almost every $x \in \mathbb{T}$, there are strictly increasing sequences of numbers $N_{j, n}$, such that for all $j=1,2,3, n \in \mathbb{N}$ we have

$$
x \in \mathcal{C}_{j}\left(\mathfrak{p}, N_{j, n}, 1 / n\right) .
$$

By the results of Propositions 4.1-4.3, this will suffice, to prove Theorem A, see §8.1 for details. 


\subsection{The $G^{\delta}$ argument}

In $\S 3.1$ we denoted by $\mathcal{B}_{\alpha} \subset \mathcal{P}$ the set of (multiplicative) coboundaries, i.e., functions $\overline{\mathfrak{p}}(x)$ such that (3.4) has a solution with a smooth transfer function $g$. We noticed after formula (3.5) that if $\overline{\mathfrak{p}} \in \mathcal{B}_{\alpha}$, then

$$
\Sigma_{x}(n)=\ln g(x+\alpha)-\ln g(x+(n+1) \alpha) .
$$

In particular, for all $n,\left|\Sigma_{x}(n)\right|$ is bounded by a constant independent of $n$, hence none of the criteria from the previous section holds for $\overline{\mathfrak{p}} \in \mathcal{B}_{\alpha}$. The advantage of coboundaries however, is that due to the simple formulas for their ergodic sums one can develop a reasonable perturbation theory and check our criteria for perturbations $\overline{\mathfrak{p}}$ of $\mathfrak{p}$ on appropriate subsets of $\mathbb{T}$.

Thus, we start by proving that the set of coboundaries are dense in $\mathcal{P}$.

Lemma 7.2. For any $\alpha \in \mathbb{R} \backslash \mathbb{Q}$, the set $\mathcal{B}_{\alpha}$ of smooth multiplicative coboundaries is dense in $\mathcal{P}$ for the $C^{\infty}$ topology.

Similarly, any $\mathfrak{p} \in C^{\infty}(\mathbb{T},(0,1)) \cap \mathcal{P}^{c}$ can be approached in the $C^{\infty}$ topology by $\overline{\mathfrak{p}}$ such that for $\overline{\mathfrak{q}}=1-\overline{\mathfrak{p}}$ and some constant $c \neq 0$, there exists $\psi \in C^{\infty}(\mathbb{T}, \mathbb{R})$ such that

$$
\ln \overline{\mathfrak{q}}(x)-\ln \overline{\mathfrak{p}}(x)=c+\psi(x+\alpha)-\psi(x) .
$$

Proof. Let $\alpha \in \mathbb{R} \backslash \mathbb{Q}$. By truncating the Fourier series of $\ln (\mathfrak{q} / \mathfrak{p})$ it is possible to approximate it in the $C^{\infty}$ topology by coboundaries of the form $\psi(\cdot)-\psi(\cdot+\alpha)$ where $\psi \in C^{\infty}(\mathbb{T}, \mathbb{R})$. Hence $F(\cdot)=g(\cdot) / g(\cdot+\alpha)$ where $g=e^{\psi}$ can be made arbitrary close to $\mathfrak{q} / \mathfrak{p}$. Now define $\overline{\mathfrak{p}}=1 /(1+F)$ and observe that $\overline{\mathfrak{p}}$ approximates $\mathfrak{p}$ and $\overline{\mathfrak{p}} \in \mathcal{B}_{\alpha}$.

The case $\mathfrak{p} \in C^{\infty}(\mathbb{T},(0,1)) \cap \mathcal{P}^{c}$ is treated similarly except that $\ln (\mathfrak{q} / \mathfrak{p})$ is approximated by $c+\psi(\cdot)-\psi(\cdot+\alpha)$ with $c \neq 0$.

To prove Theorem 7.1, we will construct explicit sequences of functions $e_{n}$, numbers $N_{j, n}$ and sets $U_{j, n}$ such that the following statement holds true:

\section{Proposition 7.3. For any Liouville number $\alpha$ there exists}

$\square$ A strictly increasing sequence of integers $q_{n}$,

$\square$ An explicit sequence of $C^{\infty}$ functions $e_{n}$ satisfying $\left|e_{n}\right|_{C^{n-1}}<1 / n$ (see $\$ 7.2 .3$ ),

$\square$ For every $j \in\{1,2,3\}$, a sequence of numbers $N_{j, n}$,

$\square$ For every $j \in\{1,2,3\}$, a sequence of sets $U_{j, n}=\bigcup_{i \in\left[0, q_{n}-1\right]}\left(I_{j, n}+i / q_{n}\right) \subset \mathbb{T}$, where $I_{j, n}$ is an interval in $\left[0,1 / q_{n}\right]$ of size $\left|I_{j, n}\right|>\frac{0.01}{q_{n}}$

with the following property. For every $\overline{\mathfrak{p}} \in \mathcal{B}_{\alpha}$, for every sufficiently large $n$

$$
U_{j, n} \subset \mathcal{C}_{j}\left(\overline{\mathfrak{p}}+e_{n}, N_{j, n}, \frac{1}{n}\right)
$$

Before proving Proposition 7.3 we show how it implies Theorem 7.1.

Proof of Theorem 7.1. Fix a Liouville $\alpha \in \mathbb{R} \backslash \mathbb{Q}$. Fix any $j \in\{1,2,3\}$. Let $U_{j, n}$ and $N_{j, n}$ be as in Proposition 7.3. Denote

$$
\mathcal{R}_{j, n}=\left\{\mathfrak{p} \in \mathcal{P} \mid U_{j, n} \subset \mathcal{C}_{j}\left(\mathfrak{p}, N_{j, n}, 1 / n\right)\right\} .
$$

By the definitions of the conditions $\mathcal{C}_{j}$, the sets $\mathcal{R}_{j, n}$ are open. Lemma 7.2 and Proposition 7.3 show that for any $m \in \mathbb{N}$ the set $\bigcup_{n \geq m} \mathcal{R}_{j, n}$ is dense. Hence the set

$$
\mathcal{R}_{j}=\bigcap_{m \in \mathbb{N}} \bigcup_{n \geq m} \mathcal{R}_{j, n}
$$


is a dense $G_{\delta}$ set (in $C^{r}$ topology for any $r \in \mathbb{N}$ ).

Observe now that for $\mathfrak{p} \in \mathcal{R}_{j}$ there exists a strictly increasing sequence $l_{n}$ such that $\mathfrak{p} \in \mathcal{R}_{j, l_{n}}$. Recall that $U_{j, l_{n}}$ has Lebesgue measure larger than 0.01 for every $l_{n}$. Moreover, up to extracting a subsequence we may assume that $q_{l_{n+1}} \gg q_{l_{n}}$, so that

$$
\lambda\left(U_{j, l_{n}} \cap \bigcap_{i=1}^{n-1} U_{j, l_{i}}^{c}\right) \geq \frac{1}{2} \lambda\left(U_{j, l_{n}}\right) \cdot \lambda\left(\bigcap_{i=1}^{n-1} U_{j, l_{i}}^{c}\right) .
$$

Now, an enhanced version of the Borel-Cantelli Lemma (see [15, Chapter IV]) states that if events $C_{n}$ are such that for each $k \geq 1$

$$
\sum_{n=k}^{\infty} \mathbb{P}\left(C_{n} \bigcap_{j=k}^{n-1} C_{j}^{c}\right)=+\infty
$$

then with probability 1 , infinitely many of those events occur. We thus conclude that a.e. $x$ belongs to infinitely many $U_{j, l_{n}}$, thus to infinitely many $\mathcal{C}_{j}\left(\mathfrak{p}, N_{j, l_{n}}, 1 / n\right)$. In conclusion, the set $\mathcal{R}=\bigcap_{j=1}^{3} \mathcal{R}_{j}$ satisfies the property required in Theorem 7.1.

The rest of Section 7 is devoted to the proof of Proposition 7.3.

\subsection{Perturbation of a smooth coboundary. The main construction}

\subsubsection{Coboundaries}

Given a smooth coboundary $\overline{\mathfrak{p}} \in \mathcal{B}_{\alpha}$ with a transfer function $g(x)$, for $M \in \mathbb{N}$, let $\bar{\Sigma}_{x}(M)$ be the ergodic sum of $\overline{\mathfrak{p}}$ defined by formula (3.5) with $\mathfrak{p}$ replaced by $\overline{\mathfrak{p}}$. Thus, for all $k \leq k^{\prime}$ and all $M \in \mathbb{N}$ we denote:

$$
\begin{aligned}
& \bar{\Sigma}_{x}(M)=\ln g(x+\alpha)-\ln g(x+(M+1) \alpha), \\
& \bar{\Sigma}_{x}\left(k, k^{\prime}\right)=\ln g(x+(k+1) \alpha)-\ln g\left(x+\left(k^{\prime}+1\right) \alpha\right), \\
& A:=\ln \|g\|+\ln \|1 / g\| .
\end{aligned}
$$

Then,

$$
\forall x \in \mathbb{T}, \forall M \in \mathbb{N}:\left|\bar{\Sigma}_{x}(M)\right| \leq A, \quad\left|\bar{\Sigma}_{x}\left(k, k^{\prime}\right)\right| \leq A,
$$

Moreover, for any smooth coboundary $\overline{\mathfrak{p}}$, there exists $0<\kappa \leq 1 / 2$ such that

$$
\kappa \leq \overline{\mathfrak{p}}(x) \leq 1-\kappa \text { for all } \quad x \in \mathrm{T} \text {. }
$$

Define

$$
K(x)=\frac{1}{\overline{\mathfrak{p}}(x)}+\frac{1}{\overline{\mathfrak{q}}(x)}
$$

and observe that

$$
K(x) \in(2,2 / \kappa]
$$

\subsubsection{The sequences $q_{n}$ and $N_{j, n}$}

Given a Liouville number $\alpha \in \mathbb{R} \backslash \mathbb{Q}$, let $q_{n}$ be a sequence of integers satisfying

$$
\eta_{n}:=\left|q_{n} \alpha\right|<q_{n}^{-n^{n}}
$$

where $|\cdot|$ denotes the closest distance to integers. Moreover, for each $n$ we will need to choose $q_{n}$ sufficiently large for our arguments to hold. 
Denote the integer closest to $q_{n} \alpha$ by $s_{n}$. In the constructions below we assume that $q_{n} \alpha>s_{n}$ for all $n$. If $q_{n} \alpha<s_{n}$, the arguments are the same up to a suitable change of signs.

Denote

$$
\begin{aligned}
& N_{n}:=\left[\left(q_{n} \eta_{n}\right)^{-1}\right] q_{n}, \\
& N_{1, n}:=\left[N_{n} / 20\right], \quad N_{2, n}:=q_{n}^{n^{5}}, \quad N_{3, n}:=N_{n},
\end{aligned}
$$

were $[a]$ stands for the integer part of $a$.

We make the following useful observation on the combinatorics of the irrational rotation $R_{\alpha}$ on the circle. The orbit of any fixed point $x$ of the circle under the rotation by $\alpha$ on $\mathrm{T}$ is essentially distributed in the following way. The points $x, x+\alpha, x+2 \alpha, \ldots x+\left(q_{n}-\right.$ 1) $\alpha$ are very close (closer than $\eta_{n}$ ) to $x, x+\frac{p_{n}}{q_{n}}, x+2 \frac{p_{n}}{q_{n}}, \ldots x+\left(q_{n}-1\right) \frac{p_{n}}{q_{n}}$. Hence, there will be one point of this $q_{n}$ piece of orbit in each basic interval $\left[k / q_{n},(k+1) / q_{n}\right], k=0, \ldots q_{n}-1$. Moreover, the first return of $x$ to its basic interval will be shifted by $\eta_{n}$. The next return will thus be shifted by one more $\eta_{n}$. Finally, the orbit $x, x+\alpha, x+2 \alpha, \ldots, x+N_{n} \alpha$ will form an $\eta_{n}$-grid inside each basic interval, and thus in the whole circle.

\subsubsection{The functions $e_{n}$}

In this section, the names of functions with the shortest period 1 are marked with a tilde, while $\frac{1}{q_{n}}$-periodic functions have no tilde in their name.

Let $\widetilde{e}_{n}(x) \in C^{\infty}$ be a 1-periodic function satisfying $\int_{\mathbb{T}} \widetilde{e}_{n}(x) d x=0$ and such that

$$
\widetilde{e}_{n}(x)= \begin{cases}\sin 8 \pi x & \text { for } x \in\left[-\frac{1}{2}+\frac{1}{n^{2}},-\frac{3}{8}-\frac{1}{n^{2}}\right] \cup\left[-\frac{3}{8}+\frac{1}{n^{2}},-\frac{1}{4}-\frac{1}{n^{2}}\right] \cup \\ & {\left[\frac{1}{4}+\frac{1}{n^{2}}, \frac{3}{8}-\frac{1}{n^{2}}\right] \cup\left[\frac{3}{8}+\frac{1}{n^{2}}, \frac{1}{2}-\frac{1}{n^{2}}\right],} \\ 0 & \text { for } x \in\left[-\frac{1}{4}, \frac{1}{4}\right], \\ \text { increasing } \quad \text { on the intervals }\left[-\frac{1}{2},-\frac{1}{2}+\frac{1}{n^{2}}\right],\left[-\frac{1}{4}-\frac{1}{n^{2}},-\frac{1}{4}\right],\left[\frac{1}{4}, \frac{1}{4}+\frac{1}{n^{2}}\right], \\ & {\left[\frac{1}{2}-\frac{1}{n^{2}}, \frac{1}{2}\right],} \\ \text { decreasing } & \text { on the intervals }\left[-\frac{3}{8}-\frac{1}{n^{2}},-\frac{3}{8}+\frac{1}{n^{2}}\right],\left[\frac{3}{8}-\frac{1}{n^{2}}, \frac{3}{8}+\frac{1}{n^{2}}\right], \\ 0 \text { and } \infty \text {-flat } & \text { at } x= \pm \frac{3}{8} .\end{cases}
$$

Observe that $\widetilde{e}_{n}$ is also flat at $\pm \frac{1}{4}$ since it is smooth. Figure 2 represents the function $\widetilde{e}_{n}$.

The idea is to perturb a given smooth coboundary $\overline{\mathfrak{p}}$ by a function of the form $q_{n}^{-n} \widetilde{e}_{n}\left(q_{n} x\right)$ to produce the desired behavior of the walk. For each $n$ we will choose $q_{n}$ satisfying (7.2) and sufficiently large. In particular, although the $C^{k}$ norms of $\widetilde{e}_{n}$ may grow fast as $n$ grows, we can still guarantee that $\left\|q_{n}^{-n} \widetilde{e}_{n}\left(q_{n} x\right)\right\|_{C^{n-1}}<\frac{1}{n}$.

A small problem is that the perturbed function $\mathfrak{p}(x)=\overline{\mathfrak{p}}(x)+q_{n}^{-n} \widetilde{e}_{n}\left(q_{n} x\right)$ may not satisfy the symmetry condition (1.3). Below we modify $\widetilde{e}_{n}(x)$ in order to assure condition (1.3) for $\mathfrak{p}$. Let $\widetilde{e}_{n}^{+}(x)$ and $\widetilde{e}_{n}^{-}(x)$ be the positive and negative parts of $\widetilde{e}_{n}(x)$ :

$$
\widetilde{e}_{n}^{+}(x)=\left\{\begin{array}{ll}
\widetilde{e}_{n}(x) & \text { if } \widetilde{e}_{n}(x) \geq 0, \\
0, & \text { otherwise, }
\end{array} \quad \widetilde{e}_{n}^{-}(x)= \begin{cases}-\widetilde{e}_{n}(x) & \text { if } \widetilde{e}_{n}(x)<0, \\
0, & \text { otherwise }\end{cases}\right.
$$

For $\delta \in[-1,1]$, define $\widetilde{e}_{n, \delta}(x)$ :

$$
\widetilde{e}_{n, \delta}(x)=\left\{\begin{array}{l}
\widetilde{e}_{n}(x)+\delta \widetilde{e}_{n}^{+}(x) \text { if } \delta \in[0,1], \\
\widetilde{e}_{n}(x)+\delta \widetilde{e}_{n}^{-}(x) \text { if } \delta \in[-1,0) .
\end{array}\right.
$$

Note that, since $\widetilde{e}_{n}$ is flat at $\pm \frac{3}{8}$ and $\pm \frac{1}{4}$, where it actually changes the sign, the functions $\widetilde{e}_{n, \delta}$ are also smooth. This is the only reason why we need $\widetilde{e}_{n}$ to be flat at those points.

The following lemma introduces the function $e_{n}$ that will be the main building block of our construction. 


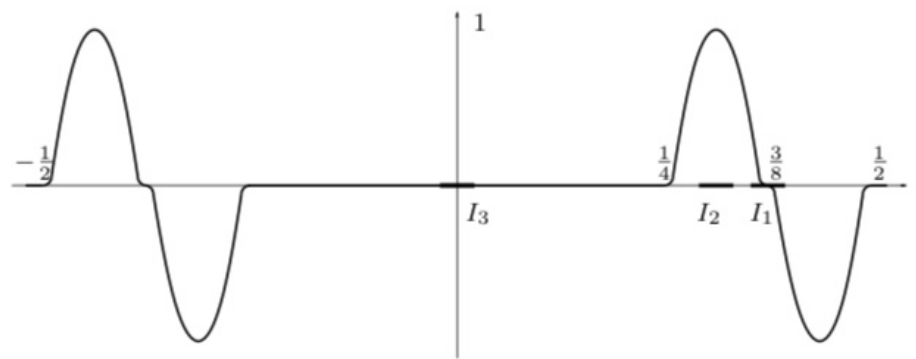

Figure 2: Graph of $\widetilde{e}_{n}(x)$. The intervals $I_{1}, I_{2}$ and $I_{3}$ are the sets for which the conditions $\mathcal{C}_{1}, \mathcal{C}_{2}$ and $\mathcal{C}_{3}$ hold (Lemmas 7.6-7.8).

Lemma 7.4. Given any $\overline{\mathfrak{p}} \in \mathcal{B}_{\alpha}$, if $q_{n}$ is sufficiently large, there exists $\delta_{n} \in\left[-\frac{1}{n}, \frac{1}{n}\right]$ satisfying

$$
\mathfrak{p}_{n}(x):=\overline{\mathfrak{p}}(x)+e_{n}(x) \in \mathcal{P} \quad \text { with } \quad e_{n}(x)=q_{n}^{-n} \widetilde{e}_{n, \delta_{n}}\left(q_{n} x\right) .
$$

Proof. In this proof, we will use the notation

$$
e_{n, \delta}(x):=q_{n}^{-n} \widetilde{e}_{n, \delta}\left(q_{n} x\right) .
$$

We are looking for $\delta \in[-1 / n, 1 / n]$ such that $\mathfrak{p}_{n}(x)$ satisfies the symmetry condition (1.3), i.e.,

$$
I_{n, \delta}:=\int_{\mathbb{T}} \ln \left(\mathfrak{p}_{n}(x)\right)-\ln \left(1-\mathfrak{p}_{n}(x)\right) d x=\int_{\mathbb{T}} \ln \left(1+\frac{e_{n, \delta}(x)}{\overline{\mathfrak{p}}(x)}\right)-\ln \left(1-\frac{e_{n, \delta}(x)}{1-\overline{\mathfrak{p}}(x)}\right) d x=0
$$

We will approximate $I_{n, \delta}$ with

$$
J_{n, \delta}:=\int_{\mathbb{T}} e_{n, \delta}(x) K(x) d x
$$

where $K$ is given by (7.1).

Claim. There exists a constant $c>0$ that does not depend on $n$ or $\delta$ such that

$$
\begin{aligned}
\left|I_{n, \delta}-J_{n, \delta}\right| & <c q_{n}^{-2 n} \\
\left|J_{n, 0}\right| & <c \frac{1}{n^{2}} q_{n}^{-n} \\
\text { For } \delta>0, \quad J_{n, \delta}-J_{n, 0} & >c \delta q_{n}^{-n} \\
\text { For } \delta<0, \quad J_{n, \delta}-J_{n, 0} & <-c \delta q_{n}^{-n}
\end{aligned}
$$

From the continuity of $I_{n, \delta}$ and $J_{n, \delta}$ in $\delta$, it follows directly from the claim that there exists $\delta \in(-1 / n, 1 / n)$ such that $I_{n, \delta}=0$.

Proof of the Claim. (7.6) follows from the fact that $\max \left|e_{n, \delta}(x)\right| \leq 2 q_{n}^{-n}$. (7.7) follows from the fact that the average of $\widetilde{e}_{n}$ is zero and from the fact that $K$ is almost constant on intervals of size $1 / q_{n}$. As for (7.8), it follows from the fact that $K>2$, and that the average of $\widetilde{e}_{n}^{+}$is larger than some positive constant independent of $n$. (7.9) is proved similarly.

Lemma 7.4 is thus proved. 


\subsubsection{The sets $U_{j, n}$}

Consider the following subintervals of $[0,1]$ :

$$
\begin{gathered}
I:=\left(-\frac{1}{200}, \frac{1}{200}\right), \\
I_{1}:=\frac{3}{8}+I, \quad I_{2}:=\frac{5}{16}+I, \quad I_{3}:=I, \\
I_{1}^{\prime}:=-\frac{3}{8}+I, \quad I_{2}^{\prime}:=-\frac{7}{16}+I,
\end{gathered}
$$

and let $I_{j, n}=I_{j} / q_{n}, I_{j, n}^{\prime}=I_{j}^{\prime} / q_{n}$ for $j=1,2,3$,

$$
U_{j, n}=\bigcup_{k=0}^{q_{n}-1}\left(I_{j, n} \cup I_{j, n}^{\prime}+\frac{k}{q_{n}}\right), \quad j=1,2, \quad U_{3, n}=\bigcup_{k=0}^{q_{n}-1} I_{3, n}+\frac{k}{q_{n}} .
$$

Notice the total measure of $U_{j, n}:\left|U_{j, n}\right|=0.02$, and $\left|U_{3, n}\right|=0.01$.

\subsection{Estimates of ergodic sums}

Recall that $\mathfrak{p}(x)=\overline{\mathfrak{p}}(x)+e_{n}(x)$ (see (7.5)), and that $N_{j, n}$ is defined by (7.3).

For $\mathfrak{p}(\cdot)$ and $\overline{\mathfrak{p}}(\cdot)$, we denote by $\Sigma_{x}(n)$ and $\bar{\Sigma}_{x}(n)$ the potential functions defined in (3.5).

The next statement represents the sums $\Sigma_{x}(M)$ for a large $M$ in the form $\Sigma_{x}(M)=$ Main $\operatorname{term}(M)+\operatorname{Rest}(M)$. Notice that the Rest $(M)$ may not be small, see (7.14) and (7.15). Nevertheless, these estimates are sufficient for the proofs in the next subsection. There we will show that, for certain values of $x$, the Rest $(M)$ is asymptotically smaller than the Main term $(M)$ provided that $M$ and $q_{n}$ are sufficiently large.

Proposition 7.5 (Main technical lemma). Given $\alpha \in \mathbb{R} \backslash \mathbb{Q}$ and a smooth coboundary $\overline{\mathfrak{p}} \in \mathcal{B}_{\alpha}$, let $A, \kappa$, and $K(x)$ be as in $\$ 7.2 .1$, and let $\widehat{K}=\int_{\mathbb{T}} K(x) d x$. Then for all $x \in \mathbb{T}$ and all $M \in\left[0, N_{n}\right]$ there exist functions $R(x, M)$ and $R^{\prime}(x, M)$ satisfying

$$
|R(x, M)|,\left|R^{\prime}(x, M)\right| \leq 4 \kappa^{-2} M q_{n}^{-2 n}
$$

such that

$$
\begin{aligned}
\Sigma_{x}(M) & =-\sum_{m=1}^{M} e_{n}(x+m \alpha) K(x+m \alpha)+\bar{\Sigma}_{x}(M)+R(x, M), \\
\Sigma_{x}(-M) & =\sum_{m=-M+1}^{0} e_{n}(x+m \alpha) K(x+m \alpha)+\bar{\Sigma}_{x}(-M)+R^{\prime}(x, M),
\end{aligned}
$$

where

$$
\begin{aligned}
& \sum_{m=1}^{M} e_{n}(x+m \alpha) K(x+m \alpha)=\widehat{K} q_{n}^{-n} N_{n} \int_{q_{n} x}^{q_{n} x+M / N_{n}} \widetilde{e}_{n, \delta_{n}}(t) d t+M o\left(q_{n}^{-n}\right), \\
& \sum_{m=-M+1}^{0} e_{n}(x+m \alpha) K(x+m \alpha)=\widehat{K} q_{n}^{-n} N_{n} \int_{q_{n} x-M / N_{n}}^{q_{n} x} \widetilde{e}_{n, \delta_{n}}(t) d t+M o\left(q_{n}^{-n}\right),
\end{aligned}
$$

Moreover,

$$
\begin{aligned}
& e_{n}(x+m \alpha) \geq 0 \text { for all } m=k, \ldots, k^{\prime} \Rightarrow \Sigma_{x}\left(k, k^{\prime}\right) \leq \bar{\Sigma}_{x}\left(k, k^{\prime}\right) \leq A \\
& e_{n}(x+m \alpha) \leq 0 \text { for all } m=k^{\prime}, \ldots, k \Rightarrow \Sigma_{x}\left(k, k^{\prime}\right) \leq \bar{\Sigma}_{x}\left(k, k^{\prime}\right) \leq A
\end{aligned}
$$


The statement of this lemma covers several different situations that will be useful in checking all the conditions $\mathcal{C}_{j}$.

Proof. Recall the notations: $\mathfrak{p}(x)=\overline{\mathfrak{p}}(x)+e_{n}(x), K=\frac{1}{\overline{\mathfrak{p}}(x)}+\frac{1}{\overline{\mathfrak{q}}(x)}$. Omitting the argument $x$, we can write:

$$
\begin{aligned}
& \ln (1-\mathfrak{p})-\ln \mathfrak{p}=\ln \left(1-\overline{\mathfrak{p}}-e_{n}\right)-\ln \left(\overline{\mathfrak{p}}+e_{n}\right)=\ln (1-\overline{\mathfrak{p}})-\ln \overline{\mathfrak{p}}+\ln \left(1-\frac{e_{n}}{1-\overline{\mathfrak{p}}}\right) \\
& -\ln \left(1+\frac{e_{n}}{\overline{\mathfrak{p}}}\right)=\ln \overline{\mathfrak{q}}-\ln \overline{\mathfrak{p}}-e_{n}\left(\frac{1}{1-\overline{\mathfrak{p}}}+\frac{1}{\overline{\mathfrak{p}}}\right)+r_{n}=\ln \overline{\mathfrak{q}}-\ln \overline{\mathfrak{p}}-e_{n} K+r_{n},
\end{aligned}
$$

where $\left|r_{n}\right| \leq 2 e_{n}^{2}\left(\bar{q}^{-2}+\bar{p}^{-2}\right) \leq\left\|K^{2}\right\| \leq 2 \kappa^{-2}$. The estimate of $\left|r_{n}\right|$ follows from the Taylor expansion of $\ln (1+y)$ for small $y$.

Now we estimate $\Sigma_{x}(M)$ for any $x \in \mathbb{T}$ and any $M>0$ :

$$
\begin{gathered}
\Sigma_{x}(M)=\sum_{m=1}^{M} \ln \left(\mathfrak{q}_{n}(x+m \alpha)\right)-\ln \left(\mathfrak{p}_{n}(x+m \alpha)\right)= \\
\sum_{m=1}^{M} \ln (\overline{\mathfrak{q}}(x+m \alpha))-\ln (\overline{\mathfrak{p}}(x+m \alpha))-\sum_{m=1}^{M} e_{n}(x+m \alpha) K(x+m \alpha)+R(x, M)
\end{gathered}
$$

and

$$
|R(x, M)| \leq\left|\sum_{m=0}^{M} e_{n}^{2}(x+m \alpha)\left(\overline{\mathfrak{q}}^{-2}(x+m \alpha)+\overline{\mathfrak{p}}^{-2}(x+m \alpha)\right)\right| \leq 4 \kappa^{-2} M q_{n}^{-2 n},
$$

since $\left\|e_{n}\right\| \leq 2 q_{n}^{-n}$ and $\overline{\mathfrak{p}}, \overline{\mathfrak{q}} \in[\kappa, 1-\kappa]$. This gives (7.12) and (7.11). The proof of (7.13) is similar.

Let us prove (7.14). By (7.2) and (7.3), we have $\eta_{n}=\left|q_{n} \alpha\right|<q_{n}^{-n^{n}}$, and $\eta_{n} \approx N_{n}^{-1}$. By definition, $e_{n}(x+\alpha)=q_{n}^{-n} \widetilde{e}_{n}\left(q_{n}(x+\alpha)\right)=q_{n}^{-n} \widetilde{e}_{n}\left(q_{n} x+\eta_{n}\right)$. For each $j \leq q_{n}$ we have:

$$
\left.\left|e_{n}(x+j \alpha)-e_{n}(x)\right|=q_{n}^{-n} \mid \widetilde{e}_{n}\left(q_{n} x+j \eta_{n}\right)-\widetilde{e}_{n}\left(q_{n} x\right)\right)\left|\leq q_{n}^{-n} \eta_{n} q_{n} \max _{\mathbb{T}}\right| \widetilde{e}_{n}(x) \mid=o\left(q_{n}^{-n}\right),
$$

since $\eta_{n}=q_{n}^{-n^{n}}$ by (7.2), and $\widetilde{e}_{n}(x)$ does not depend on $q_{n}$, see $\S 7.2 .3$ for the definition of $\widetilde{e}_{n}(x)$. Since $\alpha$ is close to $p_{n} / q_{n}$, we get:

$$
\begin{aligned}
\sum_{m=1}^{q_{n}} e_{n}(x+m \alpha) K(x+m \alpha) & =\left(e_{n}(x)+o\left(q_{n}^{-n}\right)\right) \sum_{m=1}^{q_{n}} K(x+m \alpha) \\
& =\widehat{K} q_{n}\left(e_{n}(x)+o\left(q_{n}^{-n}\right)\right) .
\end{aligned}
$$

Hence, for $M \gg q_{n}$ we have

$$
\begin{aligned}
\sum_{m=1}^{M} e_{n}(x+m \alpha) K(x+m \alpha) & =\widehat{K} q_{n} \sum_{m=1}^{M / q_{n}} e_{n}\left(x+m q_{n} \alpha\right)+M o\left(q_{n}^{-n}\right) \\
=\widehat{K} q_{n} N_{n} \sum_{m=1}^{M / q_{n}} e_{n}\left(x+m\left|q_{n} \alpha\right|\right) \eta_{n}+M o\left(q_{n}^{-n}\right) & =\widehat{K} q_{n} N_{n} \int_{x}^{x+M /\left(q_{n} N_{n}\right)} e_{n}(t) d t+M o\left(q_{n}^{-n}\right) \\
& =\widehat{K} q_{n}^{-n} N_{n} \int_{q_{n} x}^{q_{n} x+M / N_{n}} \widetilde{e}_{n, \delta_{n}}(t) d t+M o\left(q_{n}^{-n}\right) .
\end{aligned}
$$

The proof of (7.15) is similar. 
To show (7.16), notice that under the assumption $e_{n}(x+m \alpha) \geq 0$ for all $m=k, \ldots, k^{\prime}$ we have for these $m$ that $\mathfrak{p}(x+m \alpha) \geq \overline{\mathfrak{p}}(x+m \alpha)$, hence

$$
\Sigma_{x}\left(k, k^{\prime}\right)=\sum_{m=k}^{k^{\prime}} \ln \frac{\mathfrak{q}(x+m \alpha)}{\mathfrak{p}(x+m \alpha)} \leq \sum_{m=k}^{k^{\prime}} \ln \frac{\overline{\mathfrak{q}}(x+m \alpha)}{\overline{\mathfrak{p}}(x+m \alpha)}=\bar{\Sigma}_{x}(M) \leq A .
$$

Estimate (7.17) is proved in the same way.

\subsection{Proof of Proposition 7.3}

Lemma 7.6. For $n$ sufficiently large, we have:

$$
U_{1, n} \subset \mathcal{C}_{1}\left(\mathfrak{p}, N_{1, n}\right) .
$$

Proof. Fix $x \in I_{1, n}$ (the same argument holds for all $x \in U_{1, n}$ ). Then $q_{n} x$ lies in an interval of size 0.01 around the point $3 / 8$. Since $\widetilde{e}_{n, \delta_{n}}$ is smaller or equal to $\sin (8 \pi x)$ for most of the above interval, we have that for large $n$

$$
\int_{q_{n} x}^{q_{n} x+1 / 20} \widetilde{e}_{n}(t) \leq \int_{3 / 8-0.01}^{3 / 8+0.04} \sin 8 \pi t<-0.001 .
$$

By (7.14) with $M=N_{1, n}=N_{n} / 20$, we have

$$
\begin{aligned}
\sum_{m=1}^{N_{1, n}} e_{n}(x+m \alpha) K(x+m \alpha) & =\widehat{K} q_{n}^{-n} N_{1, n} \int_{q_{n} x}^{q_{n} x+1 / 20} \widetilde{e}_{n, \delta_{n}}(t) d t+N_{0} o\left(q_{n}^{-n}\right) \\
& <-0.001 \widehat{K} q_{n}^{-n} N_{1, n}
\end{aligned}
$$

Since $\left|R\left(x, N_{1, n}\right)\right| \leq 4 \kappa^{-2} N_{1, n} q_{n}^{-2 n}$, and $\kappa$ and $A$ are independent of $N_{n}$, we get from (7.12) for any $n$ sufficiently large:

$$
\Sigma_{x}\left(N_{1, n}\right)>0.001 \widehat{K} N_{1, n} q_{n}^{-n} .
$$

Recall that, by (7.2) and (7.3), $N_{n}$ is of order $q_{n}^{n^{n}}$ and $N_{1, n}=\left[N_{n} / 20\right]$. Therefore, $N_{1, n} \geq q_{n}^{n^{6}} / 40 \geq q_{n}^{6 n}$, and for sufficiently large $q_{n}$ we have $0.001 \widehat{K} \sqrt{N_{1, n}} q_{n}^{-n} \geq 1$. Hence,

$$
\Sigma_{x}\left(N_{1, n}\right)>N_{1, n}^{1 / 2} .
$$

Likewise, $\Sigma_{x}\left(-N_{1, n}\right)>N_{1, n}^{1 / 2}$.

Lemma 7.7. For $n$ sufficiently large, we have:

$$
U_{2, n} \subset \mathcal{C}_{2}\left(\mathfrak{p}, N_{2, n}, 1 / n\right) .
$$

Proof. We choose $q_{n}$ and $N_{2, n}$ satisfying (7.2) and (7.3). Let $\bar{\Sigma}_{x}(M)$ and $A$ be as in $\S$ 7.2.1; recall that $A$ only depends on $\overline{\mathfrak{p}}$. Assuming that $q_{n}$ is sufficiently large, we define

$$
L:=q_{n}^{n^{2}}>e^{e^{A}} .
$$

Since $N_{2, n}=q_{n}^{n^{5}}$ by (7.3), we have

$$
N_{2, n}=L^{n^{3}}>L^{n^{2}},
$$

and $N_{2, n} \leq e^{L^{0.1}}$, as required in $\mathcal{C}_{2}\left(\mathfrak{p}, N_{2, n}, \frac{1}{n}\right)$.

Let $x \in I_{2, n}$ (the same argument holds for all $x \in U_{2, n}$ ). Then

$$
q_{n} x \in[5 / 16-0.01,5 / 16+0.01] .
$$


By the definition of $\widetilde{e}_{n, \delta_{n}}$, for any $t \in\left[q_{n} x-0.001, q_{n} x+0.001\right]$ it holds that $\widetilde{e}_{n, \delta_{n}}(t) \geq 0.5$. Since by (7.3) we have $N_{2, n} / N_{n}<0.0001$, we get from (7.15) with $M=L<N_{2, n}$

$$
\begin{aligned}
\sum_{m=-L+1}^{0} e_{n}(x+m \alpha) K(x+m \alpha) & =\widehat{K} q_{n}^{-n} N_{n} \int_{q_{n} x-L / N_{n}}^{q_{n} x} \widetilde{e}_{n, \delta_{n}}(t) d t+N_{0} o\left(q_{n}^{-n}\right) \\
& \geq \widehat{K} 0.49 L q_{n}^{-n} .
\end{aligned}
$$

Then, since $\widehat{K}>2$, we conclude from (7.13) and (7.11) that

$$
\Sigma_{x}(-L)>0.1 L q_{n}^{-n}>\sqrt{L} .
$$

This gives $\left(\mathcal{C}_{2} a\right)$.

To verify $\left(\mathcal{C}_{2} b\right)$, notice that for $x \in I_{2, n}$ and any $m \in\left[-N_{2, n}, N_{2, n}\right]$ we have $q_{n} x+m q_{n} \alpha \in[5 / 16-0.02,5 / 16+0.02]$. Thus

$$
e_{n}(x+m \alpha)=q_{n}^{-n} \widetilde{e}_{n, \delta_{n}}\left(q_{n} x+m q_{n} \alpha\right) \geq 0 .
$$

By (7.16), we have $\left(\mathcal{C}_{2} b\right)$, i.e.,

$$
\Sigma_{x}\left(k, k^{\prime}\right) \leq A \text { for all }-N_{2, n} \leq k \leq k^{\prime} \leq N_{2, n} .
$$

To verify $\left(\mathcal{C}_{2} c\right)$, notice that for $L$ as above we have

$$
|L \alpha|=\frac{L}{q_{n}}\left|q_{n} \alpha\right|<\frac{L}{q_{n}} q_{n}^{-n^{n}}<N_{2, n}^{-n^{n} / 2} .
$$

Hence, for any $j \in[0, L-1], k \in\left[-N_{2, n}, N_{2, n}\right]$ we have $|\mathfrak{p}(x+j \alpha+k L \alpha)-\mathfrak{p}(x+j \alpha)| \leq$ $N_{2, n}^{-n^{3}}$.

Lemma 7.8. For any $n$ sufficiently large, we have:

$$
U_{3, n} \subset \mathcal{C}_{3}\left(\mathfrak{p}, N_{3, n}, 1 / n\right) .
$$

Proof. Let $x \in I_{3, n}$ be fixed (the same argument holds for all $x \in U_{3, n}$ ). Define $Q=q_{n}$, and take for the numbers $u, v, w_{ \pm}, u^{\prime}, v^{\prime}, w_{ \pm}^{\prime}\left(v, v^{\prime} \in[0.3,0.4]\right)$ to be

$$
\begin{aligned}
u=\frac{1}{4}-x q_{n}, & v=\frac{3}{8}-x q_{n}, \quad u^{\prime}=\frac{1}{4}+x q_{n}, \quad v^{\prime}=\frac{3}{8}+x q_{n}, \\
w_{ \pm}=v \pm \varepsilon, \quad w_{ \pm}^{\prime} & =v^{\prime} \pm \varepsilon,
\end{aligned}
$$

where we set $\varepsilon=\frac{1}{n}$. Assume without loss of generality that for each of the numbers introduced above, its product with $N_{n}$ is an integer that is a multiple of $q_{n}$. Let $A>0$ be as in Proposition 7.5, and assume that $q_{n}$ is sufficiently large to satisfy

$$
e^{e^{A}}<Q<N_{n}^{1 / 2} \text {. }
$$

The proof of $\left(\mathcal{C}_{3} a\right)$ is almost the same as the proof of $\left(\mathcal{C}_{1}\right)$ in Lemma 7.6. Namely, we have $x+v N_{n} \alpha=x+v / q_{n}+\mathcal{O}\left(N_{n}^{-1}\right)=3 /\left(8 q_{n}\right)+\mathcal{O}\left(N_{n}^{-1}\right)$. Hence

$$
\begin{aligned}
\Sigma_{x}\left(v N_{n}, w_{+} N_{n}\right) & =\sum_{m=1}^{\left(w_{+}-v\right) N_{n}} \ln \mathfrak{q}\left(x+\left(v N_{n}+m\right) \alpha\right)-\ln \mathfrak{p}\left(x+\left(v N_{n}+m\right) \alpha\right) \approx \\
& \sum_{m=1}^{\varepsilon N_{n}} \ln \mathfrak{q}\left(3 /\left(8 q_{n}\right)+m \alpha\right)-\ln \mathfrak{p}\left(3 /\left(8 q_{n}\right)+m \alpha\right)=\Sigma_{3 /\left(8 q_{n}\right)}\left(\varepsilon N_{n}\right) .
\end{aligned}
$$


Random walks in a Liouville quasi-periodic environment

Notice that $3 /\left(8 q_{n}\right) \in I_{1, n}$, so the analysis of the latter sum is analogous to that of Lemma 7.6. Let us repeat the argument. The sum above is estimated using (7.14). Since $1 / n^{2} \ll \varepsilon$, it follows from the definition of $\widetilde{e}_{n, \delta_{n}}$ that it is negative on most of the interval of integration $\left[\frac{3}{8}, \frac{3}{8}+\varepsilon\right]$. Moreover, on all the interval, if $\widetilde{e}_{n, \delta_{n}}(t)<0$ then $\widetilde{e}_{n, \delta_{n}}(t) \leq \sin 8 \pi t$. Thus

$$
\begin{gathered}
\sum_{m=1}^{\varepsilon N_{n}} K\left(3 /\left(8 q_{n}\right)+m \alpha\right) e_{n}\left(3 /\left(8 q_{n}\right)+m \alpha\right)=\widehat{K} q_{n}^{-n} N_{n} \int_{3 / 8}^{3 / 8+\varepsilon} \widetilde{e}_{n, \delta_{n}}(t) d t+N_{0} o\left(q_{n}^{-n}\right) \\
<\frac{\widehat{K}}{2} q_{n}^{-n} N_{n} \int_{3 / 8+\varepsilon / 2}^{3 / 8+\varepsilon} \sin (8 \pi t) d t<-0.001 \widehat{K} q_{n}^{-n} N_{n} \varepsilon^{2} .
\end{gathered}
$$

On the other hand $\left|R\left(x, \varepsilon N_{n}\right)\right| \leq 4 \kappa^{-2} \varepsilon N_{n} q_{n}^{-2 n}$, and $A$ is independent of $N_{n}$. Hence, by (7.12), and since $\widehat{K} \geq 2$

$$
\Sigma_{x}\left(v N_{n}, w_{+} N_{n}\right)>0.002 q_{n}^{-n} N_{n} \varepsilon^{2}-A-4 \kappa^{-2} \varepsilon N_{n} q_{n}^{-2 n}>N_{n}^{1 / 2}
$$

for $N_{n}$ sufficiently large. The remaining three estimates of this item are proved in the same way.

To verify $\left(\mathcal{C}_{3} b\right)$, notice that, by the definition of $u^{\prime}$ and $v$ we have:

$$
\begin{aligned}
x-u^{\prime} N_{n} \alpha & =x-u^{\prime} / q_{n}+\mathcal{O}\left(N_{n}^{-1}\right)=-1 /\left(4 q_{n}\right)+\mathcal{O}\left(N_{n}^{-1}\right), \\
x+v N_{n} \alpha & =x+v / q_{n}+\mathcal{O}\left(N_{n}^{-1}\right)=3 /\left(8 q_{n}\right)+\mathcal{O}\left(N_{n}^{-1}\right) .
\end{aligned}
$$

Hence, for all $m \in\left[-u^{\prime} N_{n}, v N_{n}\right]$ we have $e_{n}(x+m \alpha) \geq 0$. By (7.16), we have the first part of $\left(\mathcal{C}_{3} b\right)$ :

$$
\Sigma_{x}\left(k, k^{\prime}\right) \leq A \text { for all }-u^{\prime} N_{n} \leq k \leq k^{\prime} \leq v N_{n} .
$$

The second part of $\left(\mathcal{C}_{3} b\right)$ is verified in the same way using formula (7.17).

It remains to verify $\left(\mathcal{C}_{3} c\right)$. For $k \in\left[-v^{\prime} N_{n} / Q, v^{\prime} N_{n} / Q\right]$, take for $\bar{\Sigma}(k)$ the sums $\bar{\Sigma}_{x}(k)$ and let $B_{x}(M):=\Sigma_{x}(M)-\bar{\Sigma}_{x}(M)$. To verify (4.1) notice that for each $l \in\left[-N_{n} / Q, N_{n} / Q\right]$ we have

$$
|l Q \alpha|<\frac{1}{Q}
$$

Therefore, since $\bar{\Sigma}_{x}(M)=\ln g(x+\alpha)-\ln g(x+(M+1) \alpha)$

$$
\left|\bar{\Sigma}_{x}(M)-\bar{\Sigma}_{x}(M+l Q)\right| \leq 2 \frac{\|\ln g\|_{C^{1}}}{Q}<Q^{-1 / 2}
$$

if $Q$ is sufficiently large.

Next, we prove (4.2). For each $m \in\left[-u^{\prime} N_{n}, u N_{n}\right]$ we have: $x+m \alpha \in\left[-\frac{1}{4 q_{n}}, \frac{1}{4 q_{n}}\right]$, and hence $e_{n}(x+m \alpha)=0$. This implies that $\mathfrak{p}(x+m \alpha)=\overline{\mathfrak{p}}(x+m \alpha)$, and

$$
\Sigma_{x}(M)=\bar{\Sigma}_{x}(M) \text { for all } M \in\left[-u^{\prime} N_{n}, u N_{n}\right]
$$

For $m \in\left[-u^{\prime} N_{n}, v N_{n}\right]$ we have: $x+m \alpha \in\left[-\frac{1}{4 q_{n}}, \frac{3}{8 q_{n}}\right]$, and hence $e_{n}(x+m \alpha) \geq 0$. Then (7.16) implies, in particular, that that for $M \in\left[0, v N_{n}\right]$ we have

$$
\Sigma_{x}(M) \leq \bar{\Sigma}_{x}(M)
$$

For $m \in\left[-v^{\prime} N_{n}, u N_{n}\right]$ we have $e_{n}(x+m \alpha) \leq 0$, which implies the second part of (4.2) by (7.17). This completes the proof of $\left(\mathcal{C}_{3} c\right)$.

Proof of Proposition 7.3. Putting together Lemmas 7.6, 7.7, 7.8 immediately yields Proposition 7.3. 


\section{Proofs of the main theorems}

\subsection{Proof of Theorem A}

By Theorem 7.1, for any $\mathfrak{p} \in \mathcal{R}$, for almost every $x \in \mathbb{T}$, there are strictly increasing sequences of numbers $N_{j, n}$, such that for all $j=1,2,3, n \in \mathbb{N}$ we have

$$
x \in \mathcal{C}_{j}\left(\mathfrak{p}, N_{j, n}, 1 / n\right) .
$$

For $j=1$, we have that $\mathrm{p}_{i}=\mathfrak{p}(x+i \alpha)$ satisfies condition $\mathcal{C}_{1}\left(N_{1, n}\right)$. Hence, Proposition 4.1 implies Theorem $\mathrm{A}(a)$ for $T=r_{n}:=e^{\sqrt{N_{1, n} / 4}}$.

For $j=2$, we have that $\mathrm{p}_{i}=\mathfrak{p}(x+i \alpha)$ satisfies condition $\mathcal{C}_{2}\left(N_{2, n}, 1 / n\right)$.

Hence, Proposition 4.2 implies Theorem A $(b)$ for $T=s_{n}:=N_{2, n}^{5}$ and $\varepsilon_{n}=1 / n$.

For $j=3$, function $\mathrm{p}_{i}=\mathfrak{p}(x+i \alpha)$ satisfies $\mathcal{C}_{3}\left(N_{3, n}, 1 / n\right)$. The conclusion of Proposition 4.3 holds for any $T \in\left[N_{3, n}^{5}, e^{N_{3, n}^{1 / 4}}\right]$. Let us take $T=t_{n}:=N_{3, n}^{5}$. Then Proposition 4.3 implies that for some $v=v(x) \in[0.3,0.4], v^{\prime}=v^{\prime}(x) \in[0.3,0.4]$ it holds

$$
\left\{\begin{array}{l}
\mathbb{P}_{x}\left(Z_{T} \in\left[v N-\frac{N}{n}, v N+\frac{N}{n}\right]\right)>0.1, \\
\mathbb{P}_{x}\left(Z_{T} \in\left[-v^{\prime} N-\frac{N}{n}, v^{\prime} N+\frac{N}{n}\right]\right)>0.1 .
\end{array}\right.
$$

This proves Theorem $\mathrm{A}(c)$ with $b_{n}=v N_{3, n} \in\left[0.3 T^{1 / 5}, 0.4 T^{1 / 5}\right], b_{n}^{\prime}=v^{\prime} N_{3, n} \in\left[0.3 T^{1 / 5}\right.$, $\left.0.4 T^{1 / 5}\right]$, and $\varepsilon_{n}=\frac{1}{n}$.

Of course, choosing larger values for $T \in\left[N_{3, n}^{5}, e^{N_{3, n}^{1 / 4}}\right]$ allows to obtain a similar statement to (c) with $b_{n}, b_{n}^{\prime}$ of order $T^{\delta}$, for any $0<\delta<1 / 5$.

\subsection{Proof of Theorems B (a) and F (a)}

We give the proof Theorem B (a). The proof of Theorem F (a) is similar. Fix $\alpha \notin \mathbb{Q}$. Let

$$
\begin{aligned}
& \mathcal{R}_{n}=\{\mathfrak{p} \in \mathcal{P}: \exists \sigma, \exists t>n, \text { such that } \forall x \in \mathbb{T}, \forall z \in[-n, n], \\
& \left.\qquad\left|\mathbb{P}_{x}\left(Z_{t}<\sigma \sqrt{t} z\right)-\Phi(z)\right|<\frac{1}{n}\right\} .
\end{aligned}
$$

The set $\widetilde{\mathcal{R}}=\cap_{n \geq 1} \mathcal{R}_{n}$ satisfies Theorem B (a). The sets $\mathcal{R}_{n}$ are open, hence $\widetilde{\mathcal{R}}$ is a $G^{\delta}$ set.

It remains to show that $\widetilde{\mathcal{R}}$ is dense in $\mathcal{P}$. By Proposition $3.1, \widetilde{\mathcal{R}}$ contains all coboundaries. Recall that coboundaries are dense in $\mathcal{P}$ by Lemma 7.2. Hence $\widetilde{\mathcal{R}}$ is a $G^{\delta}$-dense set.

\subsection{Proof of Theorem B (b)}

Define

$$
\begin{aligned}
\mathcal{R}_{v, \varepsilon}=\{\mathfrak{p} \in \mathcal{P}: & \exists \text { open sets } \mathcal{I} \text { and } \mathcal{I}^{\prime} \text { with } \operatorname{Leb}\left(\mathcal{I}>0.001, \operatorname{Leb}\left(\mathcal{I}^{\prime}\right)>0.001\right. \\
& \text { such that } \forall\left(x, x^{\prime}\right) \in \mathcal{I} \times \mathcal{I}^{\prime}, \exists \mu(x)>v^{1-\varepsilon}, \mu\left(x^{\prime}\right)=0, \\
& \text { and for } \left.y \in\left\{x, x^{\prime}\right\}, \forall z \in[-1 / \varepsilon, 1 / \varepsilon](2.9) \text { holds with } T=v\right\}
\end{aligned}
$$

The sets $\mathcal{R}_{v, \varepsilon}$ are open, and any $\mathfrak{p} \in \hat{\mathcal{R}}:=\bigcap_{\varepsilon=\frac{1}{n}} \bigcup_{v} \mathcal{R}_{v, \varepsilon}$ satisfies Theorem B (b). Hence, it suffices to show that $\mathfrak{p}(x)=\overline{\mathfrak{p}}(x)+e_{n}(x)$, as defined in (7.5), belongs to $\mathcal{R}_{v_{n}, \frac{1}{n}}$, where 
we set $v_{n}=N_{2, n}^{5}$. For this we take $\mathcal{I}_{n}=U_{2, n}$ and apply Proposition 4.2 and get (2.9) with $\mu_{n}(x) \geq v_{n}^{1-\varepsilon_{n}}$ for every $x \in \mathcal{I}_{n}$ and every $z \in \mathbb{R}$.

On the other hand, we set $\mathcal{I}_{n}^{\prime}=U_{3, n}$ and observe that for every $t \leq v_{n}$ we have $\mathfrak{p}(x+t \alpha)=\overline{\mathfrak{p}}(x+t \alpha)$. Hence the walk for such an $x$ up to time $v_{n}$ is the same as the one with the function $\overline{\mathfrak{p}}$ that is a coboundary. Since $N_{2, n}$ can be chosen arbitrarily large as function of $\bar{p}$, we get for every $x \in \mathcal{I}_{n}^{\prime}(2.9)$ with $\mu_{n}(x)=0$.

In conclusion, the set $\mathcal{R}^{\prime}:=\overline{\mathcal{R}} \cap \hat{\mathcal{R}}$ satisfies the conditions of Theorem $\mathrm{B}$.

\subsection{Proof of Corollary C}

If the walk had an absolutely continuous stationary measure, then, by Proposition $3.1, \ln \mathfrak{q}-\ln \mathfrak{p}$ would be a smooth coboundary. Then, for any given sequence $\left\{u_{N}\right\}$ such that $u_{N} \rightarrow \infty$, we would have $\mu\left\{x:\left|\Sigma_{x}(N)\right| \geq u_{N}\right\} \rightarrow 0$ as $N \rightarrow \infty$.

However, Lemma 7.6 shows that if $\alpha$ is Liouville, then for a dense $G_{\delta}$ set of functions $\mathfrak{p} \in \mathcal{P}$ there exists a sequence $\left\{N_{j}\right\}$ such that $\left|\Sigma_{x}\left(N_{j}\right)\right|>\sqrt{N_{j}}$ for a set of $x$ of measure 0.01 . A contradiction.

\subsection{Proof of Theorem E}

Define

$$
\begin{aligned}
\mathcal{A}_{m, n}=\{\alpha \in \mathbb{R}: & \forall \mathfrak{p} \in \mathcal{P} \exists \sigma \text { such that } \forall x \in \mathbb{T}, \forall z \in[-n, n], \\
& \left.\left|\mathbb{P}_{x}\left(Z_{t}<\sigma \sqrt{t} z\right)-\Phi(z)\right|<\frac{1}{n} \text { for all } t \in\left[m, e^{m}\right]\right\} .
\end{aligned}
$$

The set $\mathcal{A}=\cap_{n \geq 1} \cup_{m \geq 1} \mathcal{A}_{m, n}$ satisfies the conclusion of the theorem. The sets $\mathcal{A}_{m, n}$ are open hence $\mathcal{A}$ is a $G^{\delta}$ set.

By [25], $\mathcal{A}$ contains the Diophantine numbers. Hence $\mathcal{A}$ is a $G^{\delta}$-dense set.

\subsection{Proof of Theorem F (b)}

Let $\lambda(x)=\frac{\mathfrak{q}(x)}{\mathfrak{p}(x)}$. To fix our notation, we assume that $\int \ln \lambda(x) d x=-c<0$ so that the walk tends to $+\infty$. The case $\int \ln \lambda(x) d x>0$ then follows by replacing $x$ by $-x$. We want to perturb $\mathfrak{p}$ to get the behavior of Theorem $F(b)$.

Let us first recall an important fact about the drift coefficient of an asymmetric walk in Theorem 2.3. Following [11], (see formula (1.6) and Theorem 4 of [11]), we associate to $\lambda$ a function

$$
u(x)=1+2 \sum_{k=0}^{\infty} \prod_{j=0}^{k} \lambda(x-j \alpha) .
$$

Then the drift coefficient of the asymmetric walk in Theorem 2.3 is given by the first integer $b_{n}(x)$ such that

$$
\sum_{k=0}^{b_{n}(x)} u_{\lambda}(x+k \alpha) \geq n .
$$

The next lemma on the Birkhoff sums of a trigonometric polynomials will be a useful tool in our perturbation of $\mathfrak{p}$.

Lemma 8.1. Let $d, M>0$ and $q$ be such that $q>e^{e^{d+M}}$. If $V$ is a trigonometric polynomial of degree $d$, and all the coefficients of $V$ are bounded by $M$, then for any $x \in \mathbb{T}$

$$
\left|\sum_{j=0}^{q-1} e^{V(x+j / q)}-q \int_{\mathbb{T}} e^{V(\theta)} d \theta\right|<e^{-q} .
$$


Proof. First, expand $e^{V(\cdot)}=\sum_{k=0}^{N} \frac{V^{k}}{k !}+\varepsilon_{N}$, where $N:=[2 q / \ln q]$, so that the error $\varepsilon_{N}$ is small compared to $e^{-q}$. On the other hand, the polynomials $V^{l}$ that we keep are all of degree strictly less than $q$, hence $\sum_{j=0}^{q-1} V^{l}\left(x+\frac{j}{q}\right)=q \int V^{l}$. The lemma follows.

Let us return to the proof of Theorem F (b). As in the proof of Theorem B (b), we only need to show density. Hence, by Lemma 7.2, we can start with a $\overline{\mathfrak{p}}$ such that for some $c>0$

$$
\ln \bar{\lambda}(x)=\ln \overline{\mathfrak{q}}(x)-\ln \overline{\mathfrak{p}}(x)=-c+\psi(x+\alpha)-\psi(x)
$$

where $\psi$ a trigonometric polynomial. Let $b_{n}$ be as in Theorem $\mathrm{F}$ (a), see (2.11). It is sufficient to prove that $\overline{\mathfrak{p}}$ can be perturbed into $\mathfrak{p}$ so that for an arbitrarily large $t_{n}$, and for some union of intervals $\mathcal{J}_{n}$ and $\mathcal{J}_{n}^{\prime}$ we have

(i) $\mu\left(\mathcal{J}_{n}\right)>0.8$ and $\mu\left(\mathcal{J}_{n}^{\prime}\right)>0.1$;

(ii) For $x \in \mathcal{J}_{n}$ we have $\left|b_{t_{n}}(x)-b_{n}\right|<t_{n}{ }^{1 / 4}$, and for $x \in \mathcal{J}_{n}^{\prime}$ we have $b_{t_{n}}(x)>b_{n}+t_{n}{ }^{0.9}$.

We start by computing the drift corresponding to $\overline{\mathfrak{p}}$ for the special sequence of times:

$$
t_{n}:=q_{n}^{n^{2}} \int_{\mathbb{T}} \bar{u}(\theta) d \theta, \quad n \in \mathbb{N} .
$$

Lemma 8.2. There is a constant $U$ (independent of $n$ ) such that for every $x \in \mathbb{T}$

$$
\left|\bar{b}_{t_{n}}(x)-q_{n}^{n^{2}}\right| \leq U
$$

Proof. Observe that if (8.3) holds then the function $\bar{u}$ associated to $\bar{\lambda}$ as in (8.1) can be written as

$$
\bar{u}(x)=1+2 \sum_{k=0}^{\infty} e^{-c(k+1)} e^{V_{k}(x)}
$$

where $V_{k}(x)=\psi(x+\alpha)-\psi(x-k \alpha)$.

Applying Lemma 8.1 to each term in (8.4) (note that the norm of $V_{k}$ is bounded uniformly in $k$ ), we conclude that if $q_{n}$ is sufficiently large then

$$
\left|\sum_{j=0}^{q_{n}-1} \bar{u}\left(x+\frac{j}{q_{n}}\right)-q_{n} \int_{\mathbb{T}} \bar{u}(\theta) d \theta\right|<e^{-q_{n}} .
$$

On the other hand, (7.2) tells us that there is an integer $p_{n}$ such that

$$
\left|\alpha-\frac{p_{n}}{q_{n}}\right| \leq q_{n}^{-n^{4}}
$$

Thus,

$$
\left|\sum_{j=0}^{q_{n}-1} \bar{u}(x+j \alpha)-\sum_{j=0}^{q_{n}-1} \bar{u}\left(x+\frac{p_{n} j}{q_{n}}\right)\right| \leq\|\bar{u}\|_{C^{1}} q_{n}^{1-n^{4}}
$$

Observe that

$$
\sum_{j=0}^{q_{n}-1} \bar{u}\left(x+\frac{p_{n} j}{q_{n}}\right)=\sum_{j=0}^{q_{n}-1} \bar{u}\left(x+\frac{j}{q_{n}}\right),
$$

since as $j$ changes from 0 to $q_{n-1}$ the set $p_{n} j$ goes over all possible residues $\bmod q_{n}$. Therefore, for every $x$ in $\mathrm{T}$ we have for $n$ sufficiently large: 


$$
\left|\sum_{j=0}^{q_{n}-1} \bar{u}(x+j \alpha)-q_{n} \int_{\mathbb{T}} \bar{u}(\theta) d \theta\right|<q_{n}^{-n^{3}}
$$

Dividing an orbit of length $q_{n}^{n^{2}}$ into pieces of length $q_{n}$, we obtain

$$
\left|\sum_{j=0}^{q_{n}^{n^{2}}} \bar{u}(x+j \alpha)-q_{n}^{n^{2}} \int_{\mathbb{T}} \bar{u}(\theta) d \theta\right|<q_{n}^{-n^{3} / 2} .
$$

This yields the conclusion of the lemma.

Now we let $g_{n}$ be a smooth function satisfying

(a) $\left\|g_{n}\right\|_{C^{n}} \leq 2^{-n}$;

(b) $g_{n}(\theta)=0$ for $\left\{q_{n} \theta\right\} \in[0,0.85]$;

(c) $g_{n}(\theta)=q_{n}^{-n-1}$ for $\left\{q_{n} \theta\right\} \in[0.86,0.99]$,

and let $\mathfrak{p}(\theta)=\overline{\mathfrak{p}}(\theta)+g_{n}(\theta)$. Define

$$
\mathcal{J}_{n}=\left\{x \in \mathbb{T}:\left\{q_{n} x\right\} \in[0,0.84]\right\}, \quad \mathcal{J}_{n}^{\prime}=\left\{x \in \mathbb{T}:\left\{q_{n} x\right\} \in[0.86,0.98]\right\} .
$$

We clearly have that $\mu\left(\mathcal{J}_{n}\right)>0.8$ and $\mu\left(\mathcal{J}_{n}^{\prime}\right)>0.1$, which is $(i)$. To finish, we need to prove $(i i)$.

Note that for $j \in\left[0,2 q_{n}^{n^{2}}\right]$ we have $q_{n}(x+j \alpha)=q_{n} x+j p_{n}+\mathcal{O}\left(q_{n}^{2-n^{4}}\right)$. Thus, it follows from (b) that for $x \in \mathcal{J}_{n}, u(x+j \alpha)=\bar{u}(x+j \alpha)$ for every $j \in\left[0,2 q_{n}^{n^{2}}\right]$. Hence, we get that $b_{t_{n}}(x)=\bar{b}_{t_{n}}(x)$, and by Lemma 8.2, taking $b_{n}:=q_{n}^{n^{2}}$ we have

$$
\left|b_{t_{n}}(x)-b_{n}\right| \leq U
$$

On the other hand, for $x \in \mathcal{J}_{n}^{\prime}$, we have from (c) that $u(x+j \alpha) \leq\left(1-q_{n}^{-n-2}\right) \bar{u}(x+j \alpha)$ for every $j \in\left[0,2 q_{n}^{n^{2}}\right]$. Hence,

$$
\sum_{j=0}^{b_{n}} u(x+j \alpha) \leq\left(1-q_{n}^{-n-2}\right)\left[\sum_{j=0}^{b_{n}} \bar{u}(x+j \alpha)\right]=t_{n}+\mathcal{O}(1)-\frac{t_{n}}{q_{n}^{-n-2}}<t_{n}-t_{n}^{0.95} .
$$

Therefore, for $x \in \mathcal{J}_{n}^{\prime}$ we have:

$$
\sum_{j=b_{n}+1}^{b_{n}(x)} u(x+j \alpha) \geq t_{n}^{0.95}
$$

and so $b_{n}(x)>b_{n}+\frac{t_{n}^{0.95}}{\max _{\theta} u(\theta)}>b_{n}+t_{n}{ }^{0.9}$. Together with (8.5) this shows (ii) and finishes the proof of Theorem $\mathrm{F}(\mathrm{b})$

\section{Appendix A Generic deterministic environments}

Here we prove Theorem 2.6. The main idea is the following. If we want to speed up the walk, we modify p by adding a drift away from the origin, while to slow it down we increase the drift towards the origin.

Proof of $(a)$. We will use the notations and definitions of Section 3.2. By (3.11), the recurrence holds iff $M(n) \rightarrow \pm \infty$ as $n \rightarrow \pm \infty$. The result follows since for each $R$ the condition that there is $n \in \mathbb{N}$ such that $M(n)>R$ and $M(-n)<-R$ is open and dense 
for the product topology introduced in Definition 2.5. Openness is straightforward, and to obtain the density it is enough to modify any given $\mathrm{p}$ to $\tilde{\mathrm{p}}$ satisfying

$$
\tilde{\mathrm{p}}(n)= \begin{cases}\frac{1}{3} & \text { for } n>K \\ \frac{2}{3} & \text { for } n<-K\end{cases}
$$

Proof of $(b)$. We also consider the environment given by (A1). Note that for this environment there are constants $C_{1}=C_{1}(K)$, and $C_{2}=C_{2}(K)$ such that

$$
|M(n)| \geq C_{1} e^{C_{2}|n|}
$$

Thus, for each $T$ and $r \geq 0$

$$
\mathbb{P}\left(\left|\bar{Z}_{T}\right| \geq r\right) \leq \frac{1}{C_{1} e^{C_{2} r}}
$$

It follows that for large $T,(2.14)$ is satisfied, showing the density of this condition. The openness is also clear.

Proof of $(c)$. It is sufficient to show that for each $\varepsilon$ the set of environments such that for some $T$

$$
\sup _{z}\left|\mathbb{P}\left(\frac{\bar{Z}_{T}-\frac{T}{3}}{\sqrt{\frac{8 T}{9}}} \leq z\right)-\Phi(z)\right|<\varepsilon
$$

is dense. We now modify any given environment so that $\tilde{\mathrm{p}}(n)=\frac{2}{3}$ for $|n|>K$. Then the walk spends a finite time to the left of $K$. It follows that

$$
\mathbb{P}\left(\frac{\bar{Z}_{T}-\frac{T}{3}}{\sqrt{\frac{8 T}{9}}} \leq z\right) \rightarrow \Phi(z)
$$

uniformly in $z$ as needed.

To prove part $(d)$, we modify a given environment outside $[-K, K]$ in three steps. First we take $K_{1} \gg K$ and modify p on $\left[-K_{1}, K_{1}\right] \backslash[-K, K]$ to achieve that

$$
\sum_{j=1}^{n} \ln \tilde{\mathfrak{q}}(j)-\ln \tilde{\mathfrak{p}}(j)=\sum_{j=-n+1}^{0} \ln \tilde{\mathfrak{p}}(j)-\ln \tilde{\mathfrak{q}}(j),
$$

where $\tilde{\mathrm{q}}(j)=1-\tilde{\mathrm{p}}(j)$. Next we take $K_{2} \gg K_{1}$ and let $\tilde{\mathrm{p}}(n)=\frac{1}{2}$ if $|n| \in\left[K_{1}+1, K_{2}\right]$. Finally, we let $\tilde{\mathrm{p}}(n)=\frac{1}{3}$ if $n<-K_{2}$ and $\tilde{\mathrm{p}}(n)=\frac{2}{3}$ if $n>K_{2}$. It is easy to see that, given $\varepsilon>0$, we can make $K_{1}$ and $K_{2}$ so large that

$$
1-\varepsilon<\frac{\left|M_{-}\right|}{\left|M_{+}\right|}<1+\varepsilon
$$

where $M_{+}$and $M_{-}$are defined in (3.12). Then (3.12) shows that

$$
\mathbb{P}\left(\lim _{t \rightarrow \infty} \bar{Z}_{T}=+\infty\right)=\frac{\left|M_{+}\right|}{\left|M_{+}\right|+\left|M_{-}\right|} \in\left[\frac{1}{2+\varepsilon}, \frac{1}{2-\varepsilon}\right] .
$$

The same holds for $\mathbb{P}\left(\lim _{t \rightarrow \infty} \bar{Z}_{T}=-\infty\right)$.

On the other hand, it is easy to see that

$$
\mathbb{P}\left(\frac{\bar{Z}_{T}-\frac{T}{3}}{\sqrt{\frac{8 T}{9}}} \leq z \mid \lim _{t \rightarrow \infty} \bar{Z}_{t}=+\infty\right)=\Phi(z)
$$


and

$$
\mathbb{P}\left(\frac{\bar{Z}_{T}+\frac{T}{3}}{\sqrt{\frac{8 T}{9}}} \leq z \mid \lim _{t \rightarrow \infty} \bar{Z}_{t}=-\infty\right)=\Phi(z) .
$$

It follows that for $T$ sufficiently large (2.16) is satisfied with $b(T)=\frac{T}{3}, \varepsilon(T)=T^{-1 / 3}$ proving the density of this condition.

\section{References}

[1] S. Alili Asymptotic behaviour for random walks in random environments, J. Appl. Prob. 36 (1999) 334-349. MR-1724844

[2] J. Brémont One-dimensional finite range random walk in random medium and stationary measure equation, Ann. Inst. H. Poincare Prob/Stat, 45 (2009) 70-103. MR-2500229

[3] J. Brémont Random walk in quasi-periodic random environment, Stochastics and Dynamics, 9 (2009) 47-70. MR-2502473

[4] J.-P. Conze, Y. Guivarch, Marches en milieu aleatoire et mesures quasi-invariants pour un systeme dynamique, Colloq. Math. 84/85 (2000) 457-480. MR-1784208

[5] D. Dolgopyat, I. Goldsheid Quenched limit theorems for nearest neighbour random walks in $1 D$ random environment, Comm. Math. Phys. 315 (2012) 241-277. MR-2966946

[6] D. Dolgopyat, I. Goldsheid Central Limit Theorem for recurrent random walks on a strip with bounded potential, Nonlinearity 31 (2018) 3381-3412. MR-3816760

[7] D. Dolgopyat, I. Goldsheid stationary measure for random walks on ergodic environments on a strip, Ann. Prob. 47 (2019) 2494-2528. MR-3980926

[8] D. Dolgopyat, I. Goldsheid Constructive approach to limit theorems for recurrent diffusive random walks on a strip, Asymptotic Analysis 122 (2021) 271-325. MR-4242830

[9] R. Durrett Probability: theory and examples. 4th ed. Cambridge Univ. Press, Cambridge, 2010. MR-2722836

[10] N. Enriquez, C. Sabot, L. Tournier, O. Zindy Quenched limits for the fluctuations of transient random walks in random environment on $\mathbb{Z}^{1}$, Ann. Appl. Probab. 23 (2013) 1148-1187. MR-3076681

[11] I. Ya. Goldsheid Simple transient random walks in one-dimensional random environment: the central limit theorem, Probab. Theory Related Fields 139 (2007) 41-64. MR-2322691

[12] A. O. Golosov Localization of random walks in one-dimensional random environments, Comm. Math. Phys. 92 (1984) 491-506. MR-0736407

[13] A. Gut, Stopped Random Walks, Springer Series in Oper. Res. \& Financial Engineering, 2009. MR-2489436

[14] G. Lorden, On Excess Over the Boundary, Annals of Math. Stat. 41, (1970), 520-527. MR0254981

[15] J. Neveu Bases mathématiques du calcul des probabilités, 2nd ed., Masson, Paris, 1970. MR-0272004

[16] W. Feller An introduction to probability theory and its applications. Vol. II, 2d ed. John Wiley \& Sons, New York-London-Sydney 1971 xxiv+669 pp. MR-0270403

[17] H. Kesten The limit distribution of Sinai's random walk in random environment, Phys. A 138 (1986) 299-309. MR-0865247

[18] H. Kesten, M. V. Kozlov, F. Spitzer Limit law for random walk in a random environment, Composito Math. 30 (1975) 145-68. MR-0380998

[19] J. Peterson Limiting distributions and large deviations for random walks in random environments, PhD Thesis - University of Minnesota, 2008. MR-2711962

[20] J. Peterson Quenched limits for transient, ballistic, sub-gaussian one-dimensional random walk in random environment, Ann. Inst. H. Poincare, Prob. Stat. 45 (2009) 685-709. MR2548499 
Random walks in a Liouville quasi-periodic environment

[21] J. Peterson, G. Samorodnitsky Weak quenched limiting distributions for transient onedimensional random walk in a random environment, Ann. Inst. Henri Poincaré Probab. Stat. 49 (2013) 722-752. MR-3112432

[22] J. Peterson, O. Zeitouni Quenched limits for transient zero-speed one-dimensional random walk in random environment, Ann. Prob. 37 (2009) 143-188. MR-2489162

[23] D. Revuz, M.Yor Continuous martingales and Brownian motion, 3d edition. Grundlehren der Mathematischen Wissenschaften 293 (1999) Springer Berlin, xiv+602 pp. MR-1725357

[24] Ya. G. Sinai The limiting behavior of a one-dimensional random walk in a random medium, Theory Prob. Appl. 27 (1982) 256-268. MR-0657919

[25] Ya. G. Sinai Simple random walks on tori, J. Statist. Phys. 94 (1999) 695-708. MR-1675369

[26] F. Solomon Random walks in a random environment, Ann. Probab. 3 (1975) 1-31. MR-0362503

[27] O. Zeitouni Random walks in random environment, Lecture Notes in Math. 1837 (2004) 193-312. MR-2071631

Acknowledgments. We are grateful for two anonymous referees who made numerous comments and suggestions that helped us make substantial revisions to the first version of this paper. Bassam Fayad thanks the KTH for excellent working conditions during his visit. 